\title{
Cross-relaxation and phonon bottleneck effects on magnetization dynamics in $\mathrm{LiYF}_{4}: \mathrm{Ho}^{3+}$
}

\author{
S. Bertaina, ${ }^{1,2}$ B. Barbara, ${ }^{1}$ R. Giraud,${ }^{1,3}$ B.Z. Malkin, ${ }^{4}$ \\ M.V.Vanuynin, ${ }^{4}$ A.I. Pominov, ${ }^{4}$ A.L. Stolov, ${ }^{4}$ and A.M. Tkachuk ${ }^{5}$ \\ ${ }^{1}$ Laboratoire de Magnétisme Louis Néel, CNRS, BP 166, 38042 Grenoble CEDEX-09,France \\ ${ }^{2}$ Ecole Nationale Supérieure de Physique de Grenoble,38402, St-Martin d'Hères, France \\ ${ }^{3} \mathrm{LPN}, \mathrm{CNRS}, 91460-$ Marcoussis, France \\ ${ }^{4}$ Kazan State University, Kazan 420008, Russian Federation \\ ${ }^{5}$ S.I. Vavilov State Optical Institute, St.Petersburg 199034, Russian Federation
}

(Dated: November 2, 2018)

\begin{abstract}
Frequency and dc magnetic field dependences of dynamic susceptibility in diluted paramagnets $\mathrm{LiYF}_{4}: \mathrm{Ho}^{3+}$ have been measured at liquid helium temperatures in the ac and dc magnetic fields parallel to the symmetry axis of a tetragonal crystal lattice. Experimental data are analyzed in the framework of microscopic theory of relaxation rates in the manifold of 24 electron-nuclear sublevels of the lowest non-Kramers doublet and the first excited singlet in the $\mathrm{Ho}^{3+}$ ground multiplet ${ }^{5} I_{8}$ split by the crystal field of $\mathrm{S}_{4}$ symmetry. The one-phonon transition probabilities were computed using electron-phonon coupling constants calculated in the framework of exchange charge model and were checked by optical piezospectroscopic measurements. The specific features observed in field dependences of the in- and out-of-phase susceptibilities (humps and dips, respectively) at the crossings (anti-crossings) of the electron-nuclear sublevels are well reproduced by simulations when the phonon bottleneck effect and the cross-spin relaxation are taken into account.
\end{abstract}

PACS numbers: 71.15.Ap, 71.15.Mb, 71.15.Rf, 71.20.Be, 75.10.Pq

\section{INTRODUCTION}

In 1968 Hellwege et al $\stackrel{\underline{1}}{r}$ found an unusual non monotonous behavior of the in-phase magnetic susceptibility in parallel dc and ac magnetic fields with narrow maxima at the dc field values corresponding to the crossings of electron-nuclear sublevels of the ground-state of diluted $\mathrm{Ho}^{3+}$ ions in $\mathrm{LaCl}_{3}$ at the temperature $\sim 1 \mathrm{~K}$ and frequencies of the ac-field in the range $10^{2}-5.10^{3}$ Hz. Recently, similar data were independently obtained on the dynamic susceptibility of the diluted paramagnet $\mathrm{LiYF}_{4}: \mathrm{Ho}^{3+}(0.1 \%)$ in the region of energy level crossings in Ref. 2]. This latter study of the anisotropic rareearth spin dynamics in the classical regime was an extension of sub-Kelvin magnetization measurements, previously used to reveal the role of quantum fluctuations of atomic magnets in the diluted $\mathrm{LiYF}_{4}: \mathrm{Ho}^{3+}$ system ${ }^{3}$, similarly to the phenomenon of resonant tunneling of single molecule magnets in presence of a large uniaxial anisotropy $\stackrel{4}{*}$. In both [1] and [2] the observed peculiarities of the susceptibility dynamics, measured at relatively high temperature, relate to predictions of N. Bloembergen and co-workers in their classical study of the crossrelaxation in spin systems ${ }^{5}$ : "...the susceptibility is usually plotted at constant frequency versus applied dc field. There may easily occur a maximum in this plot, because for certain values of the external field some pairs of levels ... may become nearly equidistant". The purpose of the present paper is to investigate the microscopic origin of the susceptibility in diluted $\mathrm{LiYF}_{4}: \mathrm{Ho}^{3+}$ at liquid helium temperatures, taking into account the different effects of crystal-field, electron-phonon and hyperfine interactions, as well as cross-relaxation processes. The ex- tension of this many-body classical dynamics to the lowtemperature quantum case was previously adressed in Refs. [2,3]. Nevertheless, many-spin quantum fluctuations in highly anisotropic systems, such as co-tunneling processes, have not been discussed on microscopic grounds up to now. Therefore, to go further in the analysis of dynamical magnetic hysteresis loops measured at sub-kelvin temperatures in the strongly out-of-equilibrium quantum regime ${ }^{3.6}$, a clear understanding of the classical spin dynamics in $\mathrm{LiYF}_{4}: \mathrm{Ho}^{3+}$ is required. The quasi-resonant absorption of radiofrequency $(5-10 \mathrm{MHz})$ ultrasound and ac magnetic field energy at the crossing points in the $\mathrm{CaWO}_{4}: \mathrm{Ho}^{3+}$ crystal was observed in Ref.[7], and some specific peculiarities of ${ }^{19} \mathrm{~F}$ nuclear relaxation rates at the crossing points in $\mathrm{LiYF}_{4}: \mathrm{Ho}^{3+}$ were found in Ref.[8]. All these experimental findings give evidence for essential variations of the relaxation rates in the electron-nuclear subsystem, by orders of magnitude, within the vicinity of energy level crossing points. In the present work, new measurements of frequency, temperature and dc magnetic field dependences of the dynamic susceptibility in $\mathrm{LiYF}_{4}$ single crystals containing different concentrations of $\mathrm{Ho}^{3+}$ ions have been carried out. The experimental data are analyzed in the framework of the microscopic theory of the electron-phonon interaction in a gapped titled system. The existing data on spectral properties of $\mathrm{Ho}^{3+}$ ions in $\mathrm{LiYF}_{4}{ }^{9}$ and additional piezospectroscopic studies described below allowed to obtain reliable values of electron-phonon coupling constants and rigorous estimates of relaxation rates in the manifold of lower electron-nuclear sublevels of the ground multiplet. Simulations based on the calculated values of kinetic parameters revealed remarkable differences between the com- 
puted and measured dependences of the dynamic susceptibility on the external parameters (frequency, temperature, strength of the constant magnetic field). We found it necessary to derive a more thorough theoretical approach accounting for the phonon bottleneck effect and the cross-relaxation. A self-consistent description of all specific features of the measured susceptibilities has been achieved using parameters of the phonon and magnetic subsystems which have physical meaning. The paper is arranged as follows: in the first section we derive a general expression of the dynamic susceptibility, then we describe the known spectral properties of impurity $\mathrm{Ho}^{3+}$ ions in $\mathrm{LiYF}_{4}$ and calculate electron-phonon coupling constants which are used to compute the relaxation matrix in the space of lower 24 electron-nuclear sublevels of the ground multiplet in the external magnetic field. The next section contains results of experimental studies, and in the last section we compare measured and simulated in- and out-of-phase susceptibilities.

\section{THEORY OF DYNAMIC MAGNETIC SUSCEPTIBILITY OF A DILUTED PARAMAGNET}

The dynamic susceptibility $\chi(\omega)$ of a single paramagnetic ion coupled to a phonon bath in the external magnetic field $\mathbf{B}=\mathbf{B}_{0}+\mathbf{B}_{1}(\omega, t),\left(\mathbf{B}_{1}(\omega, t)=\mathbf{B}_{1}^{0} \exp (-i \omega t)\right.$, is determined by the following expression:

$$
\chi_{\alpha \beta}(\omega)=\frac{\operatorname{Tr}\left(\rho(t) \Delta m_{\alpha}\right)}{B_{1 \beta}} .
$$

Here $\Delta \mathbf{m}=\mathbf{m}-\langle\mathbf{m}\rangle_{0} ; \mathbf{m}$ is the operator of the ion magnetic moment, and $\langle\ldots\rangle_{0}$ defines an average value, corresponding to the equilibrium single ion density matrix $\rho_{0}\left(H_{0}\right)=\mathrm{e}^{-H_{0} / k T} / \operatorname{Tr}\left(\mathrm{e}^{-H_{0} / k T}\right)$ in absence of the time dependent field. Time evolution of the density matrix $\rho(t)$ defined in the space of eigenfunctions $|k\rangle$ corresponding to eigenvalues $E_{k}$ of the Hamiltonian $H_{0}$ of the unperturbed electronic (or electron-nuclear) system can be described by the master equation for diagonal elements $\rho_{n n}=\rho_{n}$ and by simple exponential decay of non-diagonal matrix elements (validity of this "secular" approximation was discussed, in particular, in Refs. [10,11]):

$$
\begin{gathered}
\frac{\partial \rho_{n}}{\partial t}=\sum_{k} W_{n k} \rho_{k} \\
\frac{\partial \rho_{n k}}{\partial t}=-\left(\gamma_{n k}+i \omega_{n k}\right) \rho_{n k}+\frac{i}{\hbar}\left[\mathbf{m B}_{1}(\omega, t), \rho\right]_{n k} \\
\left(n \neq k, \omega_{n k}=\left(E_{n}-E_{k}\right) / \hbar\right)
\end{gathered}
$$

When working with eq.(2) and (3) we implicitly suppose that the equilibrium state is established in the bath much faster than in the electronic subsystem. Besides, we have omitted in the r.h.s. of eq.(2) the terms $-2 \operatorname{Im} \sum_{k \neq n} \mathbf{m B}_{1}(\omega, t) \rho_{k n} / \hbar$, which do not contribute to the linear response on the weak alternating field $\mathbf{B}_{1}(\omega, t)$. The off-diagonal elements of the relaxation matrix $W_{n k}=W_{k \rightarrow n}$ are the transition probabilities induced by the electron-phonon interaction. For the one-phonon transitions, $W_{m \rightarrow k}=w_{m k}\left[n\left(\omega_{m k}\right)+1\right]$ if the frequency of the transition $\omega_{m k}>0$ and $W_{m \rightarrow k}=$ $w_{m k} n\left(\omega_{k m}\right)$ if $\omega_{m k}<0$ where $w_{m k}$ is the probability of the spontaneous transition, and $n\left(\omega_{m k}\right)$ is the phonon occupation number. The diagonal element $W_{n n}=$ $-\sum_{k} W_{k n}$ determines the lifetime of the corresponding state $n$, and the coherence decay rate equals

$$
\gamma_{n k}=-\frac{1}{2}\left(W_{n n}+W_{k k}\right)+\Gamma_{n k}
$$

where the first term is caused by finite lifetimes, and $\Gamma_{n k}$ determines all additional contributions to the homogeneous broadening of the $n \rightarrow k$ transition.

Considering the energy of interaction with the timedependent field $-\mathbf{m B}_{1}(\omega, t)$ as a perturbation, we solve equations of motion in the linear approximation:

$$
\rho_{n}(t)=\rho_{0 n}+\Delta \boldsymbol{\rho}_{n}(\omega) \mathbf{B}_{1} \exp (-i \omega t)
$$

where $\Delta \boldsymbol{\rho}_{n}(0)=\rho_{0 n}\left(\mathbf{m}_{n n}-\langle\mathbf{m}\rangle_{0}\right) / k T$

Postulating that the interaction with the phonon bath tends to establish an equilibrium state at the instant value of the magnetic field, $\sum_{k} W_{n k} \rho_{0 k}\left(H_{0}-\mathbf{m B}_{1}\right)=0$, we obtain from eqs. (2) (3) and (4)

$$
\begin{gathered}
\Delta \rho_{\beta, n}(\omega)=\sum_{k p}(i \omega 1+\mathbf{W})_{n k}^{-1} W_{k p} \Delta m_{\beta, p p} \rho_{0 p} / k T \\
\Delta \rho_{\beta, n k}(\omega)=\frac{m_{\beta, n k}\left(\rho_{0 k}-\rho_{0 n}\right)}{\hbar\left(\omega_{n k}-\omega-i \gamma_{n k}\right)}
\end{gathered}
$$

The dynamic susceptibility (1) takes the form

$$
\begin{aligned}
\chi_{\alpha \beta}(\omega)= & \chi_{\alpha \beta}^{0}-\left\{i \omega \sum_{n k} \Delta m_{\alpha, n n}(i \omega 1+\mathbf{W})_{n k}^{-1}\right. \\
& \left.\times \Delta m_{\beta, k k} \rho_{0 k} / k T\right\} \\
& +\sum_{n, k \neq n} m_{\alpha, n k} m_{\beta, k n}\left(\rho_{0 k}-\rho_{0 n}\right) \\
& \times\left(\frac{1}{\hbar\left(\omega_{n k}-\omega-i \gamma_{n k}\right)}-\frac{1}{\hbar \omega_{n k}}\right)
\end{aligned}
$$

where the well known expression for the static susceptibility 


$$
\begin{aligned}
\chi_{\alpha \beta}^{0}= & \sum_{n} \Delta m_{\alpha, n n} \Delta m_{\beta, n n} \rho_{0 n} / k T \\
& +\sum_{n, k \neq n} \frac{m_{\alpha, n k} m_{\beta, k n}}{\hbar \omega_{n k}}\left(\rho_{0 k}-\rho_{0 n}\right)
\end{aligned}
$$

has been used. The first line in eq. (8) coincides with the expression presented in Ref. 12.

In the case of a finite concentration of paramagnetic ions, we have to take into account interactions between the ions (dipole-dipole, dimer or trimer exchange, virtual phonon exchange) and the finite rate of a heat flow from the phonon reservoir to the helium bath (the phonon bottleneck effect). These interactions between paramagnetic ions induce energy exchange (cross-relaxation), and the master equation (2) contains additional nonlinear terms ${ }^{5}$ :

$$
\begin{aligned}
\dot{\rho}_{n}= & \sum_{m} W_{n m} \rho_{m}+\sum_{m p l}\left[\left(W_{n p, l m}^{C R} \rho_{p} \rho_{m}-W_{p n, m l}^{C R} \rho_{l} \rho_{n}\right)\right. \\
& \left.-\left(W_{n p, l m}^{C R} \rho_{p} \rho_{m}-W_{p n, m l}^{C R} \rho_{l} \rho_{n}\right)_{a d}\right]
\end{aligned}
$$

Here $W_{n p, l m}^{C R}$ is the probability of the simultaneous transitions $m \rightarrow l$ of one ion, and $p \rightarrow n$ of another ion. According to the Fermi Golden rule, this transition probability can be written as follows 5.13 :

$$
W_{n p, l m}^{C R}=\frac{2 \pi}{\hbar}\left\langle|<n, l| H_{12}|p, m>|\right\rangle_{A v} \delta\left(\omega_{p n}-\omega_{l m}\right)
$$

where $H_{12}$ is the Hamiltonian of interaction between the ions, and \langle\rangle$_{A v}$ means a configuration averaging over the distribution of the paramagnetic ions in the crystal lattice. In the particular case of dipole-dipole interactions between rare-earth magnetic moments $\mathbf{m}=g_{J} \mu_{B} \mathbf{J}\left(\mu_{B}\right.$ is the Bohr magneton, $\mathbf{J}$ is the total angular momentum, $g_{J}$ is the Lande factor), the Hamiltonian $H_{12}$ has the form

$$
H_{12}=\frac{\left(g_{J} \mu_{B}\right)^{2}}{R^{3}}\left[\mathbf{J}_{1} \mathbf{J}_{2}-3\left(\mathbf{J}_{1} \mathbf{R}\right)\left(\mathbf{J}_{2} \mathbf{R}\right) / R^{2}\right]
$$

where $\mathbf{R}$ is the vector connecting the ions. The last term at the right-hand side of eq. (10), (...) $)_{a d}$, provides an asymptotical approach to the equilibrium distribution of populations in the electronic system isolated from the phonon bath (in adiabatic conditions) due to the crossrelaxation processes $\underline{\underline{5}}$.

Taking into account the distribution of the single ion energies, we can write the transition probability (11) in the following form:

$$
\begin{aligned}
W_{n p, l m}^{C R}= & \delta^{2} \sum_{\alpha \beta \gamma \delta} g_{\alpha \beta \gamma \delta}^{C R}\left(\omega_{p n}-\omega_{l m}\right) k_{\alpha \beta \gamma \delta} \times \\
& \left(<n\left|J_{1 \alpha}\right| p><l\left|J_{2 \beta}\right| m>\times\right. \\
& \left.<p\left|J_{1 \gamma}\right| n><m\left|J_{2 \alpha}\right| l>+c . c .\right)
\end{aligned}
$$

where $g_{\alpha \beta \gamma \delta}^{C R}(\omega)$ is the cross-relaxation line shape function, $\delta$ is the average energy of the interaction in frequency units (we can consider $\delta=\left(g_{J} \mu_{B}\right)^{2} / \hbar R_{0}^{3}$ with $R_{0}$ equal to a lattice constant as a scaling factor), and $k_{\alpha \beta \gamma \delta}$ are the average values of the corresponding dimensionless lattice factors. The Hamiltonian (12) contains only symmetrical products of the components of the angular moments, $J_{1 \alpha} J_{2 \beta}$, and in a general case there are 21 independent parameters $k_{\alpha \beta \gamma \delta}$. However, this number can be essentially diminished due to symmetry properties of a concrete lattice (similarly to the elastic compliance tensor). In particular, in a case of $\mathrm{S}_{4}$ local symmetry of paramagnetic ions, we obtain

$$
\begin{aligned}
& W_{n p, l m}^{C R} \delta^{-2}= \\
& g_{33}^{C R} k_{33}\left|J_{1 z}^{n p} J_{2 z}^{l m}\right|^{2} \\
+ & g_{11}^{C R} k_{11}\left(\left|J_{1+}^{n p} J_{2-}^{l m}+J_{1-}^{n p} J_{2+}^{l m}\right|^{2}\right) \\
+ & g_{66}^{C R} k_{66}\left(\left|J_{1+}^{n p} J_{2+}^{l m}-J_{1-}^{n p} J_{2-}^{l m}\right|^{2}\right) \\
+ & g_{12}^{C R} k_{12}\left(\left|J_{1+}^{n p} J_{2+}^{l m}+J_{1-}^{n p} J_{2-}^{l m}\right|^{2}\right) \\
+ & g_{44}^{C R} k_{44}\left[\left(\left|\left(J_{1+}^{n p}+J_{1-}^{n p}\right) J_{2 z}^{l m}+J_{1 z}^{n p}\left(J_{2+}^{l m}+J_{2-}^{l m}\right)\right|^{2}\right.\right. \\
+ & \left.\left|\left(J_{1+}^{n p}-J_{1-}^{n p}\right) J_{2 z}^{l m}+J_{1 z}^{n p}\left(J_{2+}^{l m}-J_{2-}^{l m}\right)\right|^{2}\right] \\
+ & g_{13}^{C R} k_{13}\left[J_{1 z}^{n p} J_{2 z}^{l m}\left(J_{1+}^{p n} J_{2-}^{m l}+J_{1-}^{p n} J_{2+}^{m l}\right)+c . c .\right] \\
+ & g_{16}^{C R} k_{16}\left[\left(J_{1+}^{n p} J_{2+}^{l m}-J_{1-}^{n p} J_{2-}^{l m}\right)\left(J_{1+}^{p n} J_{2+}^{m l}+J_{1-}^{p n} J_{2-}^{m l}\right)+\text { c.c. }\right]
\end{aligned}
$$

with $J^{a b}=\langle a|J| b>$. Because a similar expression may be obtained in a case of long range interactions between the paramagnetic ions through the field of elastic lattice deformations $\left(H_{12}^{d}=\sum_{p k n m} A_{p k}^{n m} C_{k}^{p}(1) C_{m}^{n}(2)\right.$ where $C_{p}^{k}(i)$ is the spherical tensor operating in the space of states of the ion $i$, and $A_{p k}^{n m}$ are the coupling constants ${ }^{14}$ )we consider seven factors $k_{a b}$ introduced in (14) as the phenomenological parameters.

If the system is not far from equilibrium, equations (10) can be linearized. We suppose that the adiabatic density matrix is characterized by a single parameter, the adiabatic temperature. The difference

$$
T^{-1}-T_{a d}^{-1}=\frac{\sum_{n} k E_{n} \Delta \boldsymbol{\rho}_{n} \mathbf{B}_{1}-T^{-1}\left\langle H_{0} \Delta \mathbf{m B}_{1}\right\rangle_{0}}{\left\langle H_{0}^{2}\right\rangle_{0}-\left\langle H_{0}\right\rangle_{0}^{2}}
$$

between the lattice and adiabatic temperatures is determined from the condition that the average values of the electronic energy $H_{0}-\mathbf{m B}_{1}$ obtained with the adiabatic density matrix and with $\rho(t)$ are equal. The master equation in the form (21) and the expression (8) for the dynamic susceptibility remain valid but with the additional contributions to the relaxation matrix

$$
\begin{aligned}
W_{n m}= & W_{m \rightarrow n} \\
& +\sum_{k} W_{n k}^{C R}\left[\delta_{k m}-\rho_{0 k} \frac{\left(E_{k}-\left\langle H_{0}\right\rangle_{0}\right)\left(E_{m}-\left\langle H_{0}\right\rangle_{0}\right)}{\left\langle H_{0}^{2}\right\rangle_{0}-\left\langle H_{0}\right\rangle_{0}^{2}}\right]
\end{aligned}
$$

where 
$W_{n m}^{C R}=\sum_{l p}\left(W_{n p, l m}^{C R} \rho_{0 p}+W_{n m, l p}^{C R} \rho_{0 p}-W_{p n, l m}^{C R} \rho_{0 n}\right),(n \neq m)$

Let us now take into account the finite relaxation rate of the phonon subsystem $\frac{15}{}$ considering equations of motion for the phonon occupation numbers along with the (2)):

$$
\begin{aligned}
\frac{d n\left(\omega_{m k}\right)}{d t}= & \frac{1}{\tau_{p h}\left(\omega_{m k}\right)}\left[n\left(\omega_{m k}\right)-n_{0}\left(\omega_{m k}\right)\right] \\
& +\frac{w_{m k} N}{P_{m k} \Delta \omega_{m k}}\left(\rho_{m}\left[n\left(\omega_{m k}\right)+1\right]-\rho_{k} n\left(\omega_{m k}\right)\right)
\end{aligned}
$$

here $n_{0}\left(\omega_{m k}\right)=\left[\exp \left(\hbar \omega_{m k} / k T\right)-1\right]^{-1}$ is the equilibrium occupation number of phonons at the resonance frequency $\omega_{m k}>0$ in the band with the width $\Delta \omega_{m k}, \tau_{p h}$ is the phonon lifetime, $N$ is the number of paramagnetic ions per unit volume, $P_{m k}$ is the density of states of resonant phonons (for the low-frequency acoustic phonons $P_{m k}=3 \omega_{m k}^{2} / 2 \pi^{2} v^{3}$, where $v$ is the average sound velocity). The stationary solution of the linearized coupled equations (2) and (17) brings about the same results as the solution of eq.(2) does (see eq.(8)), but with the renormalized transition probabilities induced by the phonon bottleneck:

$W_{m \rightarrow n}^{(r)}=W_{m \rightarrow n}\left[1+\frac{2 \pi^{2} v^{3} \tau_{p h}\left(\omega_{m n}\right) N w_{m n}\left|\rho_{0 m}-\rho_{0 n}\right|}{3 \omega_{m n}^{2} \Delta \omega_{m n}\left[1+i \omega \tau_{p h}\left(\omega_{m n}\right)\right]}\right]^{-1}$

To calculate the susceptibility, we need to know, first of all, elements of the relaxation matrix $\mathbf{W}$, i.e., the transition probabilities for each pair of states of a paramagnetic ion induced by the electron-phonon interaction. At the next step, the parameters of the cross-relaxation rates and the phonon lifetimes introduced in expressions (14) and (17), respectively, may be determined from a comparison of the calculated real and imaginary parts of the susceptibility (8) with the experimental data.

\section{SPECTRAL PROPERTIES OF ISOLATED $\mathrm{Ho}^{3+}$ IONS IN $\mathrm{LiYF}_{4}$}

The crystal lattice of $\mathrm{LiYF}_{4}$ has a space group $\mathrm{C}_{4 h}^{6}$ with the lattice constants $a=0.5164 \mathrm{~nm}, c=1.0741 \mathrm{~nm} . \underline{16}$ The $\mathrm{Ho}^{3+}$ ions substitute for $\mathrm{Y}^{3+}$ ions in the sites with the point symmetry $\mathrm{S}_{4}$, coordinates of the four nearest fluorine ions at the distance $R_{1}=0.2244 \mathrm{~nm}$ relative to the $\mathrm{Ho}^{3+}$ ion in the site (000) equal (x y z), (-x -y z), $(\mathrm{x}-\mathrm{y}-\mathrm{z}),(-\mathrm{x} \mathrm{y}-\mathrm{z})$, where $\mathrm{x}=(\mathrm{t}-1 / 2) a, \mathrm{y}=(1 / 2-\mathrm{p}) a$, $\mathrm{z}=-\mathrm{q} c$, and $\mathrm{p}=0.2817 ; \mathrm{t}=0.1645 ; \mathrm{q}=0.0813$. The next nearest fluorine ions are at the distance $R_{2}=0.2297 \mathrm{~nm}$, they form a deformed tetrahedron as well with $\mathrm{x}=\mathrm{t} a, \mathrm{y}$ $=(1 / 2-\mathrm{p}) a, \mathrm{z}=(\mathrm{q}-1 / 4) c)$.

The energy level pattern of the electronic $4 \mathrm{f}^{10}$ configuration of the $\mathrm{Ho}^{3+}$ ion in $\mathrm{LiYF}_{4}$ was studied in quite a few works $\stackrel{17,18,19}{ }$, fragments of this pattern are shown in
$\mathrm{E} / \mathrm{hc}\left(\mathrm{cm}^{-1}\right)$

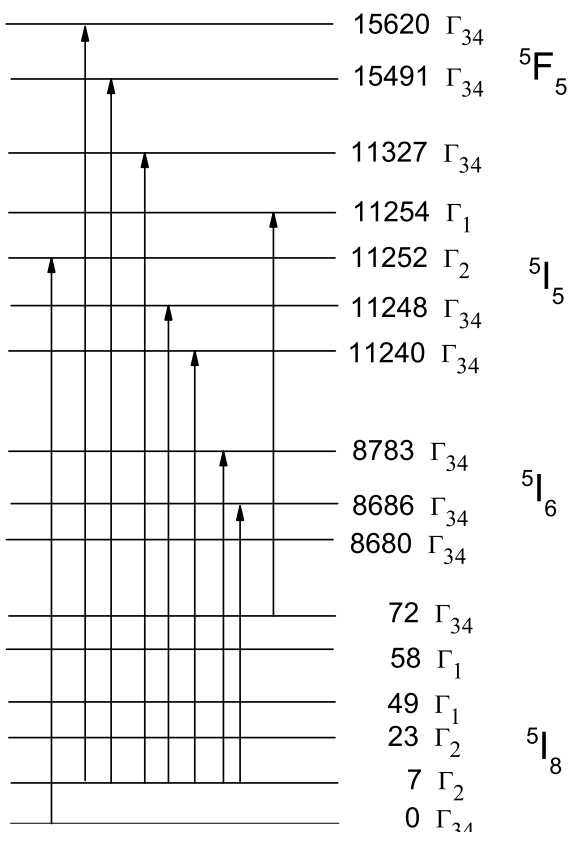

FIG. 1: Optical singlet-doublet transitions in the absorption spectrum of impurity $\mathrm{Ho}^{3+}$ ions in $\mathrm{LiYF}_{4}$ explored in the piezospectroscopic measurements.

Fig,1. The spectrum consists of singlets $\left(\Gamma_{1}\right.$ and $\left.\Gamma_{2}\right)$ and non-Kramers doublets $\left(\Gamma_{34}\right)$ corresponding to irreducible representations $\Gamma_{k}$ of the $\mathrm{S}_{4}$ group. Being interested in low temperature magnetic properties of the system, we can consider only the lower part of the energy spectrum described by the parameterized single ion Hamiltonian operating in the space of 136 products $\left|L S J J_{z}>\otimes\right| I I_{z}>$ of the electronic and nuclear spin functions (there is only one Holmium isotope ${ }^{165}$ Ho with the nuclear spin $\mathrm{I}=7 / 2$, $\mathrm{L}, \mathrm{S}, \mathrm{J}$ are the electronic orbital, spin and total angular moments, respectively, the lowest electronic multiplet is $\left.{ }^{5} \mathrm{I}_{8}\right)$ :

$$
H=H_{0}+H_{e-p h} \quad ; \quad H_{0}=H_{C F}+H_{h f}+H_{Z}+H_{S}
$$

Here

$$
\begin{aligned}
H_{C F}= & a_{2} B_{2}^{0} O_{2}^{0}+a_{4}\left(B_{4}^{0} O_{4}^{0}+B_{4}^{4} O_{4}^{4}+B_{4}^{-4} O_{4}^{-4}\right) \\
& +a_{6}\left(B_{6}^{0} O_{6}^{0}+B_{6}^{4} O_{6}^{4}+B_{6}^{-4} O_{6}^{-4}\right)
\end{aligned}
$$

is the crystal field Hamiltonian ( $B_{p}^{k}$ are the crystal field parameters, $O_{p}^{|k|}$ and $O_{p}^{-|k|}$ are the real and imaginary Stevens operators, respectively; $a_{2}=\alpha=-1 / 450$, $a_{4}=\beta=-1 / 30030, a_{6}=\gamma=-5 /\left(189.143^{2}\right)$ are the reduced matrix elements of the Stevens operators in the manifold of the pure ${ }^{5} \mathrm{I}_{8}$ states), $H_{h f}$ is the Hamiltonian of the magnetic hyperfine interaction (the electrostatic quadrupole interaction can be neglected), $H_{Z}=g_{J} \mu_{B} \mathbf{J B}$ 
is the electronic Zeeman energy in the magnetic field B; the last two terms in (19), the Hamiltonian of the electron-phonon interaction $H_{e-p h}$ and the energy of interaction with random lattice strains $H_{S}$, are specified below. As it was shown in [9], the submillimeter EPR

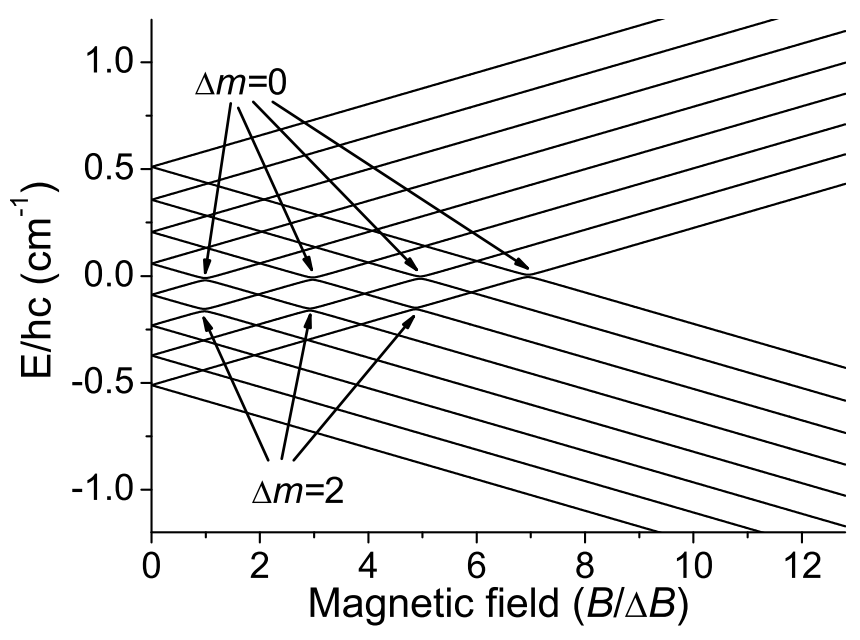

FIG. 2: Electron-nuclear sublevels of the $\mathrm{Ho}^{3+}$ ground doublet ${ }^{5} \mathrm{I}_{8}\left(\Gamma_{34}\right)$ in the magnetic field $\mathbf{B} \| c$. The magnetic field strength is given in units of $\Delta B=23.74 \mathrm{mT}$.

spectra of $\mathrm{Ho}^{3+}$ ions in $\mathrm{LiYF}_{4}$ are well described by the effective $g$-factor $g_{J}=1.21$ (slightly different from the value $5 / 4$ for the pure ${ }^{5} \mathrm{I}_{8}$ multiplet), the hyperfine constant $A=0.795 \mathrm{GHz}$, and the crystal field parameters given in Table 1.

It should be noted that this set of the crystal field parameters is related to the crystallographic system of coordinates (the quantization axis $\mathrm{z}$ is parallel to the crystal c-axis). This statement is based on the earlier studies of the piezo-spectroscopic and nonlinear Zeeman effects in $\mathrm{LiYF}_{4}: \mathrm{Tm}^{3+}$ 20,21], and the anisotropic parastriction in the concentrated paramagnets $\mathrm{LiTmF}_{4}{ }_{42}$ and $\mathrm{LiErF}_{4}{ }_{23}$. The calculated hyperfine structure of the ground electronic doublet in an external magnetic field directed along the crystal symmetry axis is shown in Fig, 2 . The spectrum consists of the two nearly equidistant groups of electron-nuclear sublevels with positive and negative slopes, respectively. The electron-nuclear sublevels intersect at the magnetic field values $B \cong\left|m^{\prime}+m\right| \Delta B$, where $m, m^{\prime}=I_{z}$ and, to first order in $A, \Delta B=A / 2 g_{J} \mu_{B}$. We can distinguish odd $\left(\mathrm{C}_{o d d},\left|m^{\prime}-m\right|=\Delta m=2 k\right)$ and even $\left(\mathrm{C}_{\text {even }},\left|m^{\prime}-m\right|=\Delta m=2 \mathrm{k}+1\right)$ crossing points at magnetic field $B=(2 n+1) \Delta B$ and $B=2 n \Delta B$, respectively, $(\mathrm{k}, \mathrm{n}=0,1,2,3)$. The mixing of the ground $\Gamma_{34}$ doublet with the excited singlets $\Gamma_{1}$ and $\Gamma_{2}$ by the magnetic hyperfine interaction opens the gaps at the odd crossing (anti-crossing) points with the maximum values for $\Delta m=2$ of an order of $0.35 \mathrm{GHz}$ (see Fig 2). However,

\begin{tabular}{|cc|c|c|c|c|}
\hline \multirow{2}{*}{$\mathrm{p}$} & $\mathrm{k}$ & \multicolumn{2}{|c|}{$B_{p}^{k}$} & $B_{p}^{k}\left(A_{g}^{1}\right)$ & $B_{p}^{k}\left(A_{g}^{2}\right)$ \\
\cline { 2 - 4 } & Exp. & Theo. & & \\
\hline & 1 & 2 & 3 & 4 & 5 \\
\hline 2 & 0 & 190.4 & 154 & 603 & -891 \\
\hline 4 & 0 & -78.2 & -89 & 125 & 718 \\
\hline 4 & 4 & -657.2 & -700 & 2397 & 1434 \\
\hline 4 & -4 & -568.6 & -613 & 3700 & 717 \\
\hline 6 & 0 & -3.2 & -2.1 & 113 & -416 \\
\hline 6 & 4 & -364.0 & -322 & 937 & -843 \\
\hline 6 & -4 & -222.3 & -265 & 1738 & -889 \\
\hline \hline \multirow{2}{*}{$\mathrm{p}$} & $\mathrm{k}$ & $B_{p}^{k}\left(B_{g}^{1}\right)$ & \multicolumn{2}{|c|}{$B_{p}^{k}\left(B_{g}^{2}\right)$} \\
\cline { 2 - 5 } & Exp. & Theo. & Exp. & Theo. \\
\hline & 6 & 7 & 8 & 9 & 10 \\
\hline 2 & 2 & 1644 & 1800 & 3814 & 3590 \\
\hline 2 & -2 & 1846 & 2070 & -836 & -1620 \\
\hline 4 & 2 & -454 & -780 & -1532 & -1980 \\
\hline 4 & -2 & 1885 & 3660 & 1424 & 1900 \\
\hline 6 & 2 & 188 & 230 & -243 & -730 \\
\hline 6 & -2 & -543 & -520 & -658 & -710 \\
\hline 6 & 6 & -858 & -740 & -1444 & -1750 \\
\hline 6 & -6 & -738 & -990 & -1245 & -2010 \\
\hline
\end{tabular}

\begin{tabular}{|c|c|c|c|c|c|}
\hline $\mathrm{p} \mathrm{k}$ & $B_{p k, 1}\left(E_{g}\right)$ & $B_{p k, 2}\left(E_{g}\right)$ & $\mathrm{p} \mathrm{k}$ & $B_{p k, 1}\left(E_{g}\right)$ & $B_{p k, 1}\left(E_{g}\right)$ \\
\hline 11 & 12 & 13 & 14 & 15 & 16 \\
\hline $\begin{array}{|ll|}2 & 1 \\
\end{array}$ & 1951 & 3595 & $\mid$\begin{tabular}{||ll}
$\mid 2$ & -1
\end{tabular} & -3595 & 1951 \\
\hline 41 & -2056 & -1996 & $4-1$ & 1996 & -2056 \\
\hline $\begin{array}{|ll|}6 & 1 \\
\end{array}$ & -1198 & -256 & 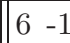 & 256 & -1198 \\
\hline $\begin{array}{|ll|}43 \\
\end{array}$ & 23372 & 16638 & $4-3$ & 16638 & -23372 \\
\hline $\begin{array}{|ll|}6 & 3 \\
\end{array}$ & 2093 & -1674 & $\mid 6-3$ & -1674 & -2093 \\
\hline 55 & -7558 & -600 & $\mid$\begin{tabular}{||ll}
6 & -5 \\
\end{tabular} & 600 & -7558 \\
\hline
\end{tabular}

TABLE I: Crystal field parameters and electron-deformation coupling constants $\left(\mathrm{cm}^{-1}\right)$

in addition to the avoided level crossings with $\Delta m=2$, gaps of comparable values $(\sim 0.28 \mathrm{GHz})$ were observed at the odd crossing points for the electron-nuclear sublevels with $\Delta m=0$ in the EPR spectra of the isotopically enriched sample ${ }^{7} \mathrm{LiYF}_{4}: \mathrm{Ho}^{3+}(0.1 \%)^{9}$. We suppose that these splittings are induced by random strains due to intrinsic lattice defects. As we shall see below, the presence of random strains is confirmed by some specific features of magnetic field dependences of the dynamic susceptibility as well, and to account for the corresponding random crystal field effects, we introduce the Hamiltonian $H_{s}=a_{2}\left(B_{2}^{2} O_{2}^{2}+B_{2}^{-2} O_{2}^{-2}\right)$ with the initial values of parameters $B_{2}^{2}=\mp 0.4525 \mathrm{~cm}^{-1} ; B_{2}^{-2}= \pm 0.4752$ $\mathrm{cm}^{-1}$ which has been already used in [9] to describe the $\Delta m=0$ anti-crossings observed in the EPR spectra of the sample isotopically enriched in ${ }^{7} \mathrm{Li}$.

At low temperatures interactions between $\mathrm{Ho}^{3+}$ ions play an important role in relaxation processes in the coupled electron-nuclear subsystem²,3,6. For a pair of distant $\mathrm{Ho}^{3+}$ ions, the energies of lower 256 electron-nuclear sub- 


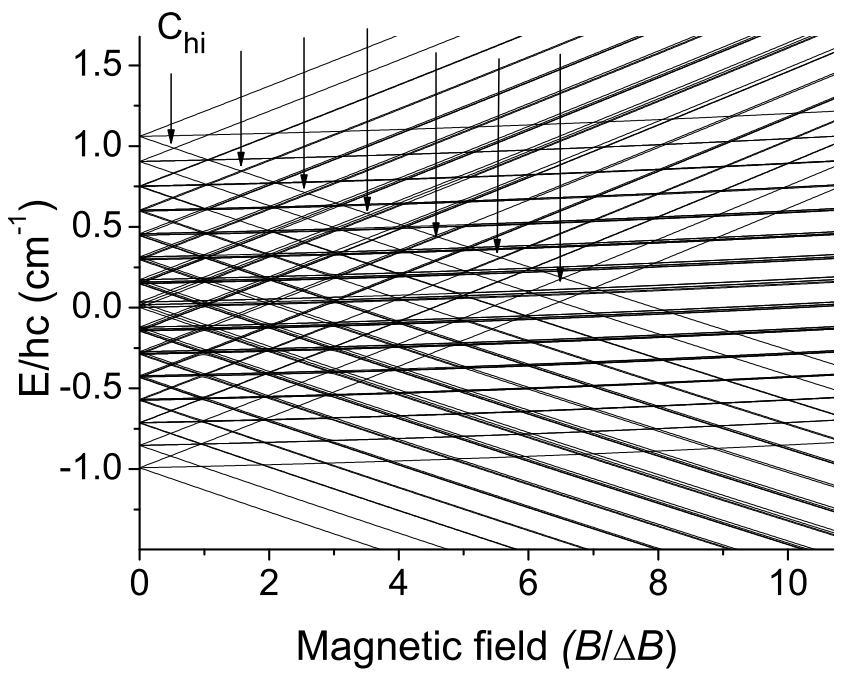

FIG. 3: Electron-nuclear sublevels of the ground state of a pair of distant holmium ions in the magnetic field $\mathbf{B} \| c$. Arrows show half-integer crossings

levels versus the magnetic field are shown in Fig. 3 . This figure presents a new plot of the sum of single ion energies related to a ground $\Gamma_{34}$ doublet where the random "rhombic" crystal field is taken into account. It is described by the Hamiltonian $H_{S}$ and mainly affects the electron-nuclear sublevels with the nuclear spin projections $m= \pm 1 / 2$. The corresponding shifts (about 0.01 $\mathrm{cm}^{-1}$ ) cause splittings of degenerate energy levels of a dimer which are concentrated close to the centre of gravity of the spectrum in Fig 3 (in particular, in zero magnetic field, 32 states have zero energy ( 8 with positive, 8 with negative, and 16 with zero slope in the magnetic field). It is well seen in Fig.3 that there are many additional crossings of the electron-nuclear sublevels in the pair spectrum as compared to the single ion spectrum (Fig,2), and the most important qualitative peculiarity is the presence of half-integer crossings $\left(\mathrm{C}_{h i}\right)$ at the magnetic field values $B \cong(n+1 / 2) \Delta B$ for $n=0,1 \ldots 6$.

Widths of transitions between the electron-nuclear sublevels of the ground doublet and the first excited singlet measured in EPR spectra are strongly dependent on the holmium concentration ${ }^{\underline{9}}$ at liquid helium temperatures. Assuming a Gaussian distribution, the inhomogeneous broadening can be defined by the FWHM (full width at half maximum) of $175 \mathrm{MHz}^{9}$. According to Ref. $[24]$, frequencies of resonance transitions of the two non-equivalent nearest neighbor fluorine nuclei in the super-hyperfine ${ }^{5} \mathrm{~F}_{5}{ }^{-5} \mathrm{I}_{8}$ spectrum equal 29.85 and 36.86 $\mathrm{MHz}$. The corresponding widths of $\mathrm{Ho}^{3+}$ energy levels due to the super-hyperfine interactions may be estimated as $130 \mathrm{MHz}$, and the total linewidths at low concentrations $(\sim 0.1 \%)$ are close to $300 \mathrm{MHz}$. The magnetic dipole-dipole interactions dominate at holmium concentrations larger than $0.5 \%$, and the observed line widths exceed $1000 \mathrm{MHz}$ in the sample containing about $1 \%$ of impurity $\mathrm{Ho}^{3+}$ ions.

\section{MODELING OF THE ELECTRON-PHONON INTERACTION AND SPIN-LATTICE RELAXATION RATES}

We shall consider interactions of impurity $\mathrm{Ho}^{3+}$ ions with acoustical phonons with energies less than $40 \mathrm{~K}$ at liquid helium temperatures (energies of optical phonons in $\mathrm{LiYF}_{4}$ are larger than $100 \mathrm{~K}^{25}$ ). The corresponding phonon wave lengths exceed essentially the lattice constants, and we can use the lattice elasticity theory to describe corresponding dynamic lattice deformations.

In the linear approximation, the Hamiltonian of the electron-phonon interaction corresponding to the modulation of the crystal field by elastic waves can be written $\operatorname{as}^{26}$

$$
\begin{aligned}
H_{e-p h}= & \sum_{\Gamma j \lambda} \sum_{p k} B_{p, \lambda}^{k}\left(\Gamma^{j}\right) e_{\lambda}\left(\Gamma^{j}\right) a_{p} O_{p}^{k} \\
& +i \sum_{\alpha} \vartheta_{\alpha}\left[H_{C F}+H_{h f}+H_{Z},\left(J_{\alpha}+I_{\alpha}\right)\right]
\end{aligned}
$$

here the first and second terms on the right describe the electron-deformational and electron-rotational interactions, respectively. Linear combinations $e_{\lambda}\left(\Gamma^{j}\right)$ of the deformation tensor components $\left(e_{\alpha \beta}\right)$ which transform according to irreducible representations of the factor group $\mathrm{C}_{4 h}$ of the lattice $\left(e\left(A_{g}^{1}\right)=e_{z z} ; e\left(A_{g}^{2}\right)=\left(e_{x x}+e_{y y}\right) / 2\right.$; $e\left(B_{g}^{1}\right)=e_{x x}-e_{y y} ; e\left(B_{g}^{2}\right)=e_{x y} ; e_{1}\left(E_{g}\right)=e_{x z} ; e_{2}\left(E_{g}\right)=$ $\left.e_{y z}\right)$ and the rotation vector $\vartheta$ are linear in the phonon annihilation $\left(a_{j q}\right)$ and creation $\left(a_{j q}^{+}\right)$operators. In particular,

$$
e_{\alpha \beta}=\sum_{q, j=j_{a c}} \frac{q}{\sqrt{N m}}[\alpha \beta, 1]\left(\frac{\hbar}{2 \omega_{j}(\mathbf{q})}\right)^{1 / 2}\left(a_{j \mathbf{q}}+a_{j-\mathbf{q}}^{+}\right)
$$

where

$$
[\alpha \beta, \sigma]=\frac{1}{2}\left(e_{\alpha}\left(j \mathbf{q}_{0}\right) q_{0 \beta}+\sigma e_{\beta}\left(j \mathbf{q}_{0}\right) q_{0 \alpha}\right), \sigma= \pm 1
$$

the sum is taken over acoustical branches $j_{a c}$ of the lattice vibrational spectrum, $N$ is the number of unit cells having the mass $m, e_{\alpha}\left(j_{a c} \mathbf{q}_{0}\right)$ are the components of the unit polarization vector in the elastic wave with the unit propagation vector $\mathbf{q}_{0}=\mathbf{q} / q$ and the frequency $\omega_{j}(\mathbf{q})$. The components of the rotation vector $\theta_{\gamma}$ are given by the same expression (22) where $[\alpha \beta,-1]$ is substituted for $[\alpha \beta, 1]$ and $\alpha \neq \beta \neq \gamma$. Parameters of the electrondeformational interaction

$$
\left\{\begin{array}{l}
B_{p}^{k}\left(A_{g}^{1}\right)=B_{p, z z}^{k} \\
B_{p}^{k}\left(A_{g}^{2}\right)=B_{p, x x}^{k}+B_{p, y y}^{k} \\
B_{p}^{k}\left(B_{g}^{1}\right)=\left(B_{p, x x}^{k}-B_{p, y y}^{k}\right) / 2 \\
B_{p}^{k}\left(B_{g}^{2}\right)=2 B_{p, x y}^{k} \\
B_{p, 1}^{k}\left(E_{g}\right)=2 B_{p, x z}^{k} \\
B_{p, 2}^{k}\left(E_{g}\right)=2 B_{p, y z}^{k}
\end{array}\right.
$$


can be computed if the crystal field parameters $B_{p}^{h}$ for an impurity rare earth ion are known as explicit functions of coordinates $X_{\lambda L, \alpha}$, of the host lattice ions (the vector $\mathbf{R}_{\lambda L}$ defines the equilibrium position of an ion $\lambda$ in the unit cell $L$ ). The corresponding calculations were carried out in the framework of the exchange charge model of the crystal field ${ }^{26}$. Parameters of the crystal field Hamiltonian are represented by a sum of two terms

$$
\begin{aligned}
B_{p}^{k}= & e^{2} K_{p}^{k} \sum_{\lambda L}\left[-Z_{\lambda}\left(1-\sigma_{p}\right) \frac{<r^{p}>}{R_{\lambda L}^{p+1}}\right. \\
& \left.+\frac{2(2 p+1)}{7 R_{\lambda L}} S_{p}\left(R_{\lambda L}\right)\right] O_{p}^{k}\left(\vartheta_{\lambda L}, \varphi_{\lambda L}\right)
\end{aligned}
$$

related to the electrostatic fields of point lattice ions with the effective charges $e Z_{\lambda}$ and "exchange" charges at the neighbor ions proportional to the quadratic forms of the overlap integrals of the 4 f-electron and ligand wave functions $S_{s}=<4 f 0\left|n^{\prime \prime} s 0>, S_{\sigma}=<4 f 0\right| n^{\prime \prime} p 0>$, $S_{\pi}=<4 f 1 \mid n^{\prime \prime} p 1>$ (only outer filled $n^{\prime \prime} s^{2}$ and $n^{\prime \prime} p^{6}$ electronic shells of ligand ions are considered):

$$
S_{p}(R)=G_{s} S_{s}^{2}(R)+G_{\sigma} S_{\sigma}^{2}(R)+\left(2-\frac{p(p+1)}{12}\right) G_{\pi} S_{\pi}^{2}(R)
$$

Here $K_{p}^{k}$ are the numerical coefficients ${ }^{26}, \sigma_{p}$ are the shielding constants, $\left\langle r^{p}\right\rangle$ are the moments of the $4 \mathrm{f}$ electron charge density, $O_{p}^{k}(\vartheta, \varphi)$ are the homogeneous Stevens polynomials formed from the direction cosines of ligand radius-vectors (the spherical coordinates of a ligand are $R, \vartheta, \varphi$ relative to the rare earth ion at the origin). Calculations were performed with $\sigma_{2}=0.579$, $\sigma_{4}=\sigma_{6}=0^{27},\left\langle r^{2}\right\rangle=0.695,\left\langle r^{4}\right\rangle=1.219,\left\langle r^{6}\right\rangle$ $=4.502$ (atomic units) $)^{28}$, the lattice sums were computed by the Ewald method, the dependences of the overlap integrals (computed with the radial wave functions from Refs. 29, 30]) on the distance $R$ (in atomic units) between the ions were approximated by functions $S_{0} \exp (-b R)$ where $S_{0}=2.2298,0.6535,1.3239 ; b=1.2554,0.88259$, 1.1596 for $s, \sigma$ and $\pi$ bonds, respectively. The values of the model parameters $G_{s}=G_{\sigma}=5.6, G_{\pi}=2.85$ were obtained from fitting the calculated crystal field energies to the experimental data. The model results in the set of crystal field parameters (Table 1, column 3) which are well comparable with those found from the analysis of the optical and EPR spectra (Table 1, column 2) $\stackrel{9}{\text {. The }}$ coupling constants $B_{p, \lambda}^{k}\left(\Gamma^{j}\right)$ in the Hamiltonian of the electron-deformational interaction presented in Table 1 (columns 4,5,8,10,12,13,15,16) were calculated using the same parameters of the model.

Now we have in hands all the data which are necessary to calculate the single phonon transition probabilities introduced in section 2 :

$$
\begin{aligned}
& W_{m \rightarrow f}=\frac{\omega_{m f}^{3}}{\pi \hbar \rho} \sum_{j=j_{a c}} \int \frac{\sin \theta d \theta d \varphi}{4 \pi v_{j}^{5}(\theta \varphi)} \times \\
& \left|\sum_{\alpha \beta}<f\right| \sum_{p k} B_{p, \alpha \beta}^{k}[\alpha \beta,+1] a_{p} O_{p}^{k} \\
& +i \hbar \omega_{f m}[\alpha \beta,-1] \sum_{\gamma} \varepsilon_{\alpha \beta \gamma}\left(J_{\gamma}+I_{\gamma}\right)|m>|^{2}\left(n_{0}\left(\omega_{m f}\right)+1\right)
\end{aligned}
$$

Here $\varepsilon_{\alpha \beta \gamma}$ is the unit antisymmetric tensor, $v_{j}(\theta \varphi)$ is the sound velocity in the direction of the phonon wave vector $\mathbf{q}$ determined by the angular coordinates $\theta$ and $\varphi$. The integrals $\sum_{j=j_{\alpha}} \int[\alpha \beta, \sigma]\left[\gamma \delta, \sigma^{\prime}\right] d \Omega / 4 \pi v_{j \alpha}^{5}$ were computed in Ref. 31].

The analysis of the magnetic field and temperature dependences of the relaxation rates involved the numerical diagonalization of the matrix of the Hamiltonian $H_{0}$ (see eq.(19) ) for the fixed values of the magnetic field (the matrix was constructed in the space of 136 functions $\left|{ }^{5} I_{8}, J J_{z}>\otimes\right| I I_{z}>$ of the lowest multiplet), calculations of transition probabilities for each pair of the electronnuclear sublevels using the corresponding eigenfunctions of $H_{0}$, and the diagonalization of the relaxation matrix W. Fig团 (a) shows the magnetic field dependences at the temperature $2 \mathrm{~K}$ of the eigenvalues of the relaxation matrix defined in the subspace of sublevels of the ground doublet. In this case transitions are mainly induced by the dynamic deformations of $B_{g}$ symmetry between the sublevels with the same projections of the nuclear spin. However, mixing of wave functions of the ground doublet with the wave functions of the excited singlets allows transitions with the nuclear spin reverting as well. At high magnetic fields $(B>0.18 \mathrm{~T})$, there are two sets of solutions each consisting of eight branches corresponding to allowed (fast relaxation rates exceeding $10^{3} s^{-1}$ ) and forbidden (slow relaxation rates less than $10^{2} s^{-1}$ ) transitions (of course, there is always a zero solution corresponding to the equilibrium state of the system). At lower magnetic fields we see specific variations of four upper branches at the $\Delta m=0$ anti-crossings and of three lower branches at the $\Delta m=2$ anti-crossings pointed in Fig 2 The additional narrow peaks superimposed on the broad peaks are caused by more narrow $\Delta m=-2$ anti-crossings which are shifted from the $\Delta m=2$ anticrossings along the magnetic field axis because the spectrum is only approximately equidistant.

Fig 4(b) demonstrates a remarkably different behavior of the considered single ion relaxation rates at the same temperature, $2 \mathrm{~K}$, when the transitions between the first excited singlet and the ground doublet are taken into account (these transitions are induced by lattice deformations of $E_{g}$ symmetry). There are again two sets of fast and slow relaxation rates, but all rates become larger by one-two orders of magnitude, the dips at the $\Delta m=0$ anti-crossings disappear, and six additional peaks in the 


\section{EXPRIMENTAL}

\section{A. Piezospectroscopic measurements}

To determine parameters of the Hamiltonian (21) corresponding to the interaction of the $\mathrm{Ho}^{3+}$ ion with lattice strains of $B_{g}$-symmetry, we measured splittings of nine $\Gamma_{34}$ doublets (see Fig.11) induced by the uniaxial stress applied in the basis plane of the lattice. The sample $\mathrm{LiYF}_{4}$ containing 1 at.\% of holmium was grown by the
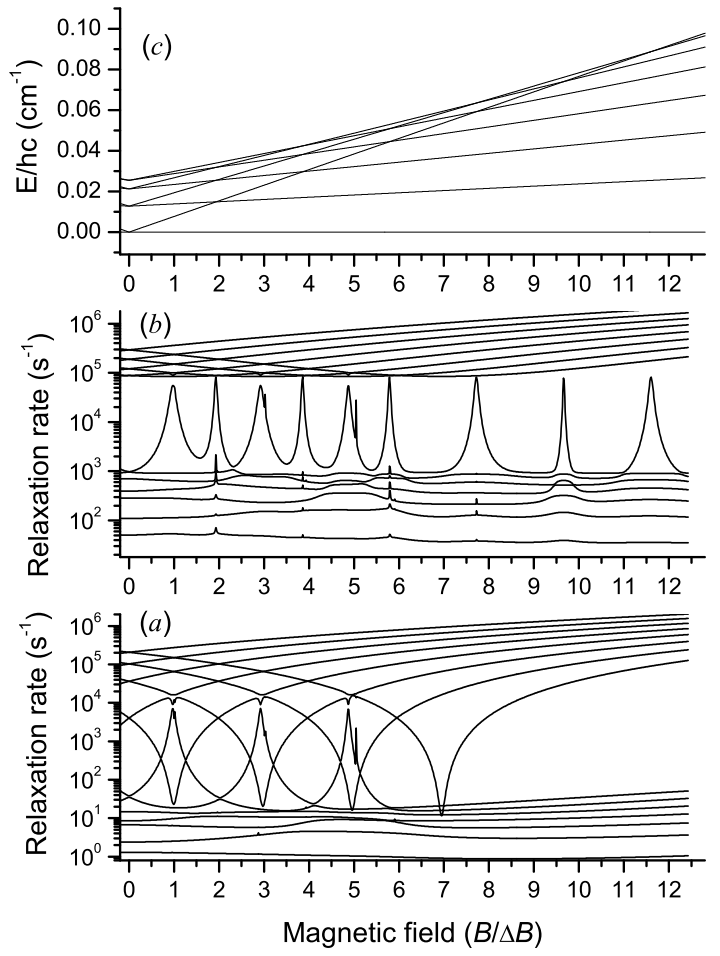

FIG. 4: Electron-phonon relaxation rates of a single $\mathrm{Ho}^{3+}$ ion in the phonon bath at the temperature $2 \mathrm{~K}$. (a) - calculated eigenvalues of the relaxation matrix defined in the space of 16 electron-nuclear sublevels of the ground doublet, (b) - calculated lower relaxation rates corresponding to one-phonon transitions between all sublevels of the ground doublet and the first singlet, (c) - relative energies of the electron-nuclear sublevels of the first excited singlet in the magnetic field $\mathbf{B} \| c$.

lower branches are observed due to the anti-crossings between the sublevels of the first excited singlet $\Gamma_{2}$ which are shown in Fig 4 (c). It follows from the results of calculations of the relaxation rates at liquid helium temperatures that the frequency dependence of the out-of-phase susceptibility of $\mathrm{Ho}^{3+}$ ions should exhibit a maximum at frequencies close to $10^{5}-10^{6} \mathrm{~Hz}$ contrary to the earlier observations ${ }^{2}$ of this maximum at frequencies below $10^{3} \mathrm{~Hz}$. This contradiction stimulated us to test the theory by direct measurements of the electron-phonon coupling constants in the piezospectroscopic experiments described in the following section.
Bridgman-Stockebarger method and oriented with the Xray diffractometer. Experiments were performed at temperatures $4.2 \mathrm{~K}$ and $77 \mathrm{~K}$, the pressure $p$ up to $220 \mathrm{MPa}$ was supplied along three different directions in the (001) plane. Splittings of the optical lines corresponding to singlet-doublet transitions in the $\sigma$ polarized absorption spectra (the wave vector of the incident light was parallel to the c-axis) were measured directly or from the linear dichroism signals ${ }^{20}$.

The uniaxial pressure in the plane (001) at the angle $\varphi$ relative to the $a$ axis induces the following nonzero components of the deformation tensor:

$$
\left\{\begin{array}{l}
e\left(A_{g}^{1}\right)=-S_{13} p \\
e\left(A_{g}^{2}\right)=-\left(S_{11}+S_{12}\right) p / 2 \\
e\left(B_{g}^{1}\right)=-\left[\left(S_{11}-S_{12}\right) \cos 2 \varphi+S_{16} \sin 2 \varphi\right] p \\
e\left(B_{g}^{2}\right)=-\left(2 S_{16} \cos 2 \varphi+S_{66} \sin 2 \varphi\right) p / 4
\end{array}\right.
$$

Here $S_{i j}$ are the components of the elastic compliance tensor of $\mathrm{LiYF}_{4}$ measured in Ref. [32], in particular, $S_{11}-$ $S_{12}=18.6, S_{16}=8.01, S_{66}=57.7\left(10^{-6} / M P a\right)$. In the linear approximation, the corresponding splitting of the doublet with the wave functions $\mid \Gamma_{34} \pm>$ equals

$$
\begin{aligned}
\Delta\left(\Gamma_{34}, \varphi\right) & =2\left|\left\langle\Gamma_{34}+\left|\sum_{p k, i=1,2} B_{p}^{k}\left(B_{g}^{i}\right) e\left(B_{g}^{i}\right) a_{p} O_{p}^{k}\right| \Gamma_{34}-\right\rangle\right| \\
& =p\left(C+D \cos \left(4 \varphi-\varphi_{0}\right)\right)^{1 / 2}
\end{aligned}
$$

Using the data obtained at three different values of the angle $\varphi$ (see Table 2), we determined for each doublet the corresponding deformation potentials $C$ and $D$ and the directions $\varphi_{0}$ of the petals of the four-fold rosettes (28). Then the set of 16 electron-deformation parameters $B_{p}^{k}\left(B_{g}^{i}\right)(p k \rightarrow 22,2-2,42,4-2,62,6-2,6$ $6,6-6)$ was varied starting from the values obtained in the framework of the exchange charge model (Table 1, columns 8,10 ) to obtain the best estimations of the measured splittings. Results of the fitting procedure are given in Table 1 (columns 7,9), and the calculated and experimental splittings are compared in Table 2 . We had only 18 independent nonlinear equations for 16 variables. It should be noted that the positive direction of the b-axis relative to the a-axis was unknown, and signs of angles $\varphi$ were checked in the fitting procedure. Possible errors in the measured splittings (in particular, due to random internal strains and errors in the orientation of the sample) are rather large $\left( \pm 0.15 \mathrm{~cm}^{-1} / 100 \mathrm{MPa}\right)$, the linear 


\begin{tabular}{|c|cc|c|cc|c|}
\hline \multirow{N}{*}{} & \multirow{2}{*}{$\begin{array}{c}\text { Energy of } \Gamma_{34} \\
\left(\mathrm{~cm}^{-1}\right)\end{array}$} & \multicolumn{3}{|c|}{ Splitting $\Delta(\varphi)\left(\mathrm{cm}^{-1} / 100 \mathrm{MPa}\right)$} \\
\cline { 4 - 7 } & & $\varphi=5^{\circ}$ & $\varphi=30.7^{\circ}$ & $\varphi=45^{\circ}$ \\
\hline 1 & ${ }^{5} \mathrm{I}_{8}$ & 0 & $0.33(0.43)$ & 0.67 & $(0.63)$ & $0.53(0.53)$ \\
2 & ${ }^{5} \mathrm{I}_{8}$ & 72 & $1.12(1.50)$ & $1.50(1.98)$ & $1.14(1.57)$ \\
3 & ${ }^{5} \mathrm{I}_{6}$ & 8686 & $0.62(0.37)$ & 0.71 & $(0.35)$ & $0.38(0.21)$ \\
4 & ${ }^{5} \mathrm{I}_{6}$ & 8783 & $1.90(1.43)$ & $2.20(1.93)$ & $2.00(1.56)$ \\
5 & ${ }^{5} \mathrm{I}_{5}$ & 11240 & $0.34(0.49)$ & 0.44 & $(0.45)$ & $0.34(0.30)$ \\
6 & ${ }^{5} \mathrm{I}_{5}$ & 11248 & $0.43(0.53)$ & $0.50(0.78)$ & $0.53(0.68)$ \\
7 & ${ }^{5} \mathrm{I}_{5}$ & 11327 & $1.45(1.33)$ & $1.95(1.77)$ & $1.60(1.42)$ \\
8 & ${ }^{5} \mathrm{~F}_{5}$ & 15491 & $0.52(0.72)$ & 0.67 & $(0.90$ & $0.62(0.85)$ \\
9 & ${ }^{5} \mathrm{~F}_{5}$ & 15620 & $1.01(1.02)$ & $1.18(1.33)$ & $0.82(1.05)$ \\
\hline
\end{tabular}

TABLE II: Splittings of non-Kramers doublets in $\mathrm{LiYF}_{4}: \mathrm{Ho}^{3+}$ (1\%) crystals axially compressed in the basis plane. Results of calculations are in brackets.

approximation for some closely spaced doublets (having numbers 3,5,6 in Table 2) is not valid, and the parameters $B_{p}^{k}\left(B_{g}^{i}\right)$ have not been determined unambiguously. However, as it is seen in Table 1, the final set of parameters $B_{p}^{k}\left(B_{g}^{i}\right)$ does not differ qualitatively from the starting values. Thus, the model used in calculations of the parameters of the electron-phonon interaction has been additionally approved. A possible error in the estimations of the matrix elements of electronic operators in the Hamiltonian of electron-phonon interaction (21) which we need to calculate the transition probabilities between the sublevels of the ground doublet does not exceed $24 \%$.

\section{B. Measurements of the dynamic susceptibility}

Earlier the dynamic susceptibility of diluted $\mathrm{LiY}_{1-c} \mathrm{Ho}_{c} \mathrm{~F}_{4}$ crystals with holmium concentrations $c=46 \%, 16.7 \%$ and $4.5 \%$ was studied in Ref. 33-35. at low temperatures with main attention for the spin-glass behavior in a random dipolar-coupled Ising magnet.

In the present work, the dynamic susceptibility of highly diluted $\mathrm{LiY}_{1-c} \mathrm{Ho}_{c} \mathrm{~F}_{4}$ single crystals was measured with a conventional SQUID magnetometer at frequencies from 20 to $1200 \mathrm{~Hz}$ in the temperature range $1.75-4$ $\mathrm{K}$. Three samples with dimensions of $4 \times 4 \times 10 \mathrm{~mm}^{3}$ were studied in collinear ac- and dc-magnetic fields parallel to the c-axis of amplitude of $410^{-4} \mathrm{~T}$ and up to $0.25 \mathrm{~T}$ respectively. The holmium concentrations in these samples $(c=0.104 \% ; 0.157 \% ; 0.27 \%)$ were determined by a comparison of the measured low frequency susceptibility with the calculated single ion static susceptibility. The in- and out-of-phase susceptibility was measured in a more extended dc field, frequencies, temperatures and concentrations, than in Ref [2], where the results of measurements of the in- and out-of-phase susceptibilities at frequencies 163 and $800 \mathrm{~Hz}$ in the sample with holmium concentration $\mathrm{c}=0.04 \%$ were published.

The results presented in Figs 5 to 7 show the non

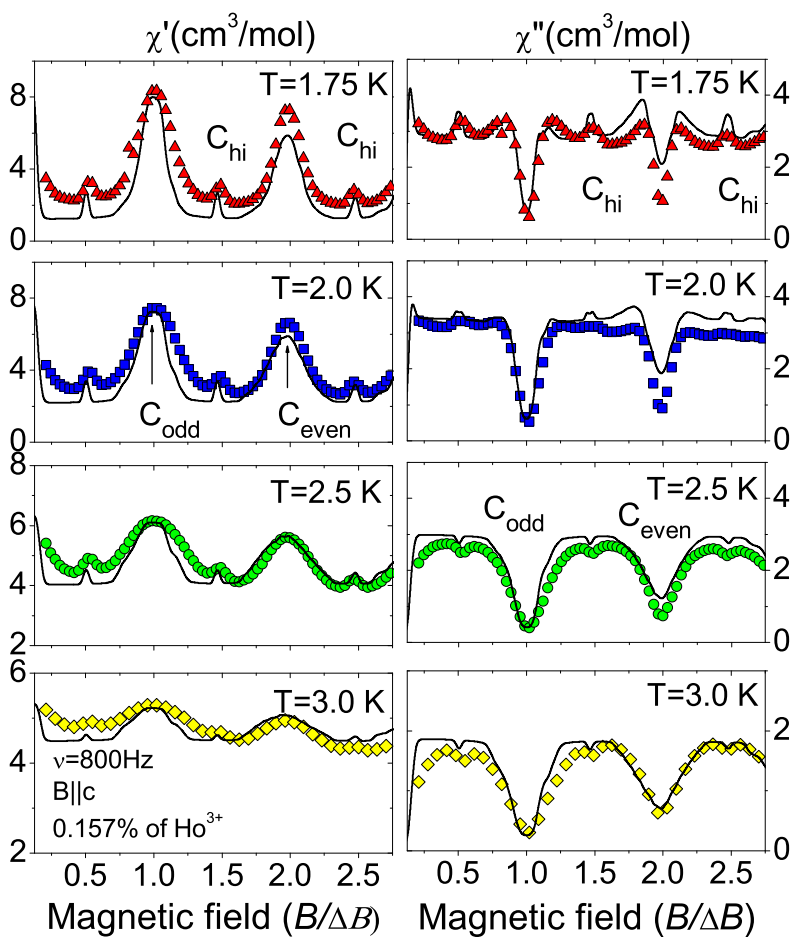

FIG. 5: (Color online) Measured and simulated magnetic field dependences of the ac-susceptibility in $\mathrm{LiYF}_{4}: \mathrm{Ho}^{3+}(0.157 \%)$ at different temperatures $(\nu=800 \mathrm{~Hz}, \mathbf{B} \| c)$.

monotonous behavior with well pronounced peaks and dips at different crossing points of the electron-nuclear energy levels. In particular, the out-of-phase susceptibility measured in the vicinity of the crossing points for two holmium concentrations show an inversion of the sign of $\mathrm{C}_{h i}$ peaks (dips) with respect to the background (Fig 6). Fig 8 presents the frequency dependences of the in- and out-of-phase susceptibilities at three different temperatures while the temperature and concentration shifts of the out-of-phase susceptibility with frequency are given in Fig9. Note that in both cases the dc magnetic field was taken in between two single-ion electron-nuclear crossing points $(38.5 \mathrm{mT}$, corresponding to $1.62 \Delta B$ ). The temperature dependences of the inand out-of-phase susceptibilities at different ac frequencies are shown in Fig 10.

\section{SIMULATIONS OF THE DYNAMIC SUSCEPTIBILITY IN LiYF $4: \mathrm{Ho}^{3+}$ AND DISCUSSION}

The experimental data presented in Figs 5 [10 are accompanied by results of the corresponding simulations. Calculations of the susceptibilities involved the numerical diagonalization of the single ion Hamiltonian $H_{0}$ (see eq.(19)) in the space of 136 electron-nuclear states of the lowest ${ }^{5} I_{8}$ multiplet for the fixed values of the external magnetic field, the computing of the matrix elements of 

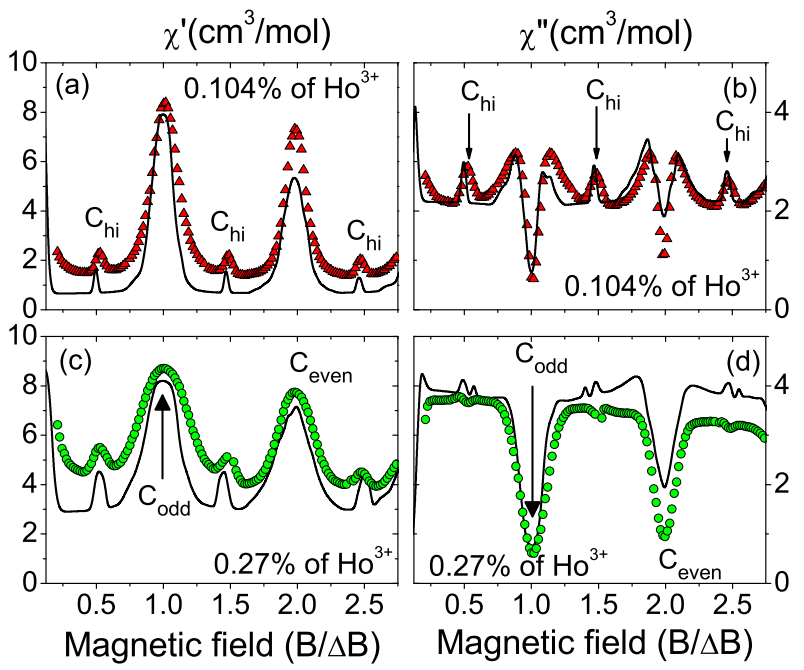

FIG. 6: (Color online) . Measured and simulated magnetic field dependences of the ac-susceptibility at the temperature $1.75 \mathrm{~K}$ in $\mathrm{LiYF}_{4}: \mathrm{Ho}^{3+}$ samples with different holmium concentrations $\mathrm{x}(\mathrm{a}, \mathrm{b}-\mathrm{x}=0.104 \%, \mathrm{c}, \mathrm{d}-\mathrm{x}=0.27 \%, \nu=800$ $\mathrm{Hz}, \mathbf{B} \| c)$.
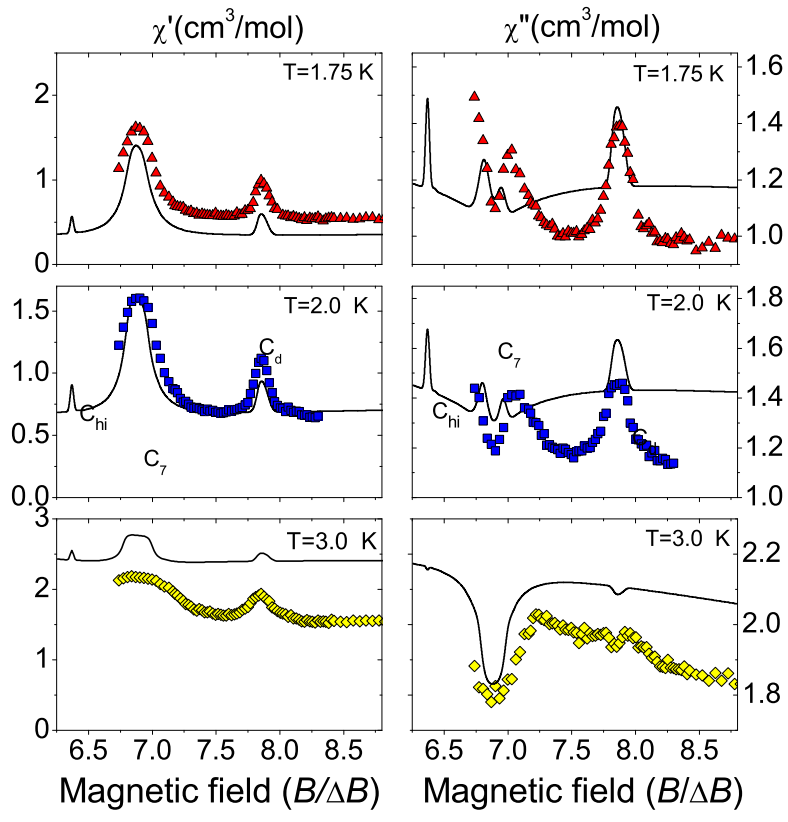

FIG. 7: (Color online). Measured and simulated magnetic field dependences of the ac-susceptibility in $\mathrm{LiYF}_{4}: \mathrm{Ho}^{3+}$ $(0.104 \%)$ in the region of high-field crossings at different temperatures $(\nu=1200 \mathrm{~Hz}, \mathbf{B} \| c)$.

electronic operators which are necessary to construct the relaxation matrices (14) and (27), and at the last step $\chi^{\prime}(\omega)=\operatorname{Re} \chi_{z z}(\omega)$ and $\chi^{\prime \prime}(\omega)=\operatorname{Im} \chi_{z z}(\omega)$ were obtained at different frequencies and temperatures using the expression (8).

First of all, let us consider the frequency dependences

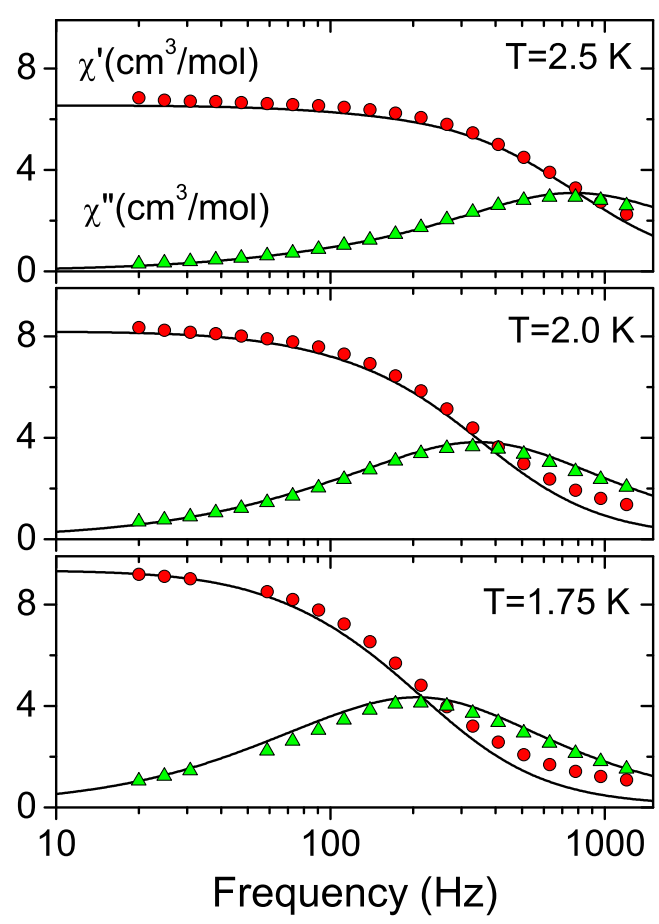

FIG. 8: (Color online). Measured and simulated frequency dependences of the dynamic susceptibility in $\mathrm{LiYF}_{4}: \mathrm{Ho}^{3+}(0.104$ $\%)$ at different temperatures $(\mathbf{B} \| c, \mathrm{~B}=38.5 \mathrm{mT})$.

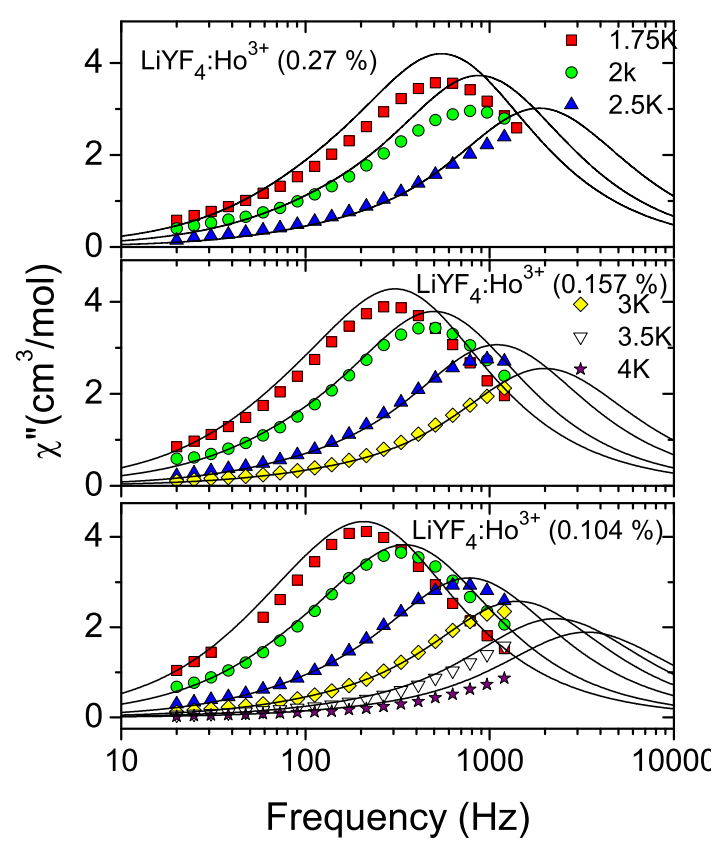

FIG. 9: (Color online). Measured and simulated frequency dependences of the out-of-phase susceptibility in $\mathrm{LiYF}_{4}: \mathrm{Ho}^{3+}$ samples at different temperatures $(\mathbf{B} \| c, \mathrm{~B}=38.5 \mathrm{mT})$. 


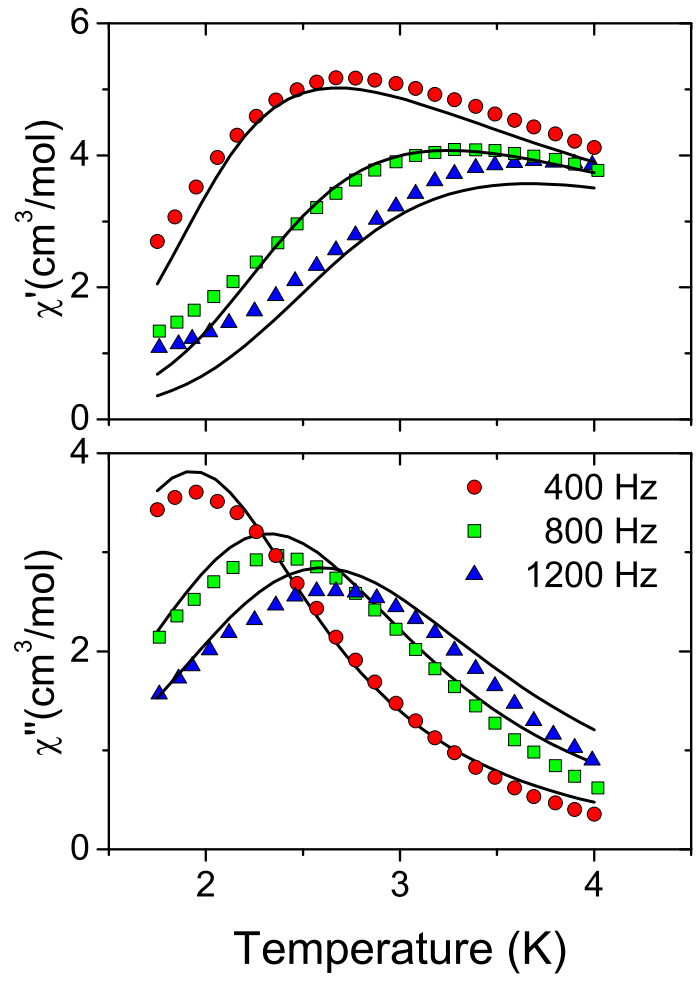

FIG. 10: (Color online). Measured (symbols) and simulated (solid curves) temperature dependences of the dynamic susceptibility in $\mathrm{LiYF}_{4}: \mathrm{Ho}^{3+}(0.104 \%)$ at different frequencies of the ac magnetic field $(\mathbf{B} \| c, \mathrm{~B}=38.5 \mathrm{mT})$.

of the out-of-phase susceptibilities in Fig. 9 measured at the dc magnetic field value of $1.62 \Delta B$, which is far enough from the neighboring single-ion crossing points. The maximum of the out-of-phase susceptibility shifts to higher frequencies with the increasing temperature. These shifts give evidence for the increasing relaxation rates induced by the electron-phonon interaction. However, using the one-phonon transition probabilities calculated with the parameters of the electron-deformational interaction from Table 1, we found the corresponding maxima at the frequencies $10^{4}-10^{5} \mathrm{~Hz}$ (see Fig 4 with the plotted relaxation times), which are about two orders of magnitude higher than the experimental values. Even more, Fig 9 also shows a concentration dependence of the maximum of the out-of-phase susceptibility, while it is clear that single-ion electron-phonon interaction cannot produce any dependence of the relaxation rate on the concentration of relaxing ions. Thus, to fit the simulated frequency dependences to the experimental curves, we had to take into account the phonon bottleneck effect. The concentration dependence of the renormalized transition probability (18) is determined by a factor $K_{m k}=\tau_{p h}\left(\omega_{m k}\right) N / \Delta \omega_{m k}$. Using the average sound velocity $v=3.10^{3} \mathrm{~m} / \mathrm{s}^{32}$, the number of paramagnetic ions per unit volume $N=2 c / V$ (here $V$ is the unit cell volume which contains two $\mathrm{Y}^{3+}$ sites accessible for holmium ions, $c$ is the concentration determined from measurements of $\left.\chi^{\prime}(\omega \rightarrow 0)\right)$, and the widths of spectral distributions of the resonant phonons $\Delta \omega_{m k}=2 \gamma_{m k}=$ $300 \mathrm{MHz}$ according to the EPR data on the widths of the singlet-doublet transitions 9 , we found it necessary to introduce two different phonon lifetimes independent on temperature to describe the experimental data for the sample with the concentration $c=0.104 \%$ (see Figs 8 and 9). Namely, we used $\tau_{p h}(\omega)=\tau_{g} \sim 1 \mu \mathrm{s}$ for resonant phonons with frequencies corresponding to transitions between the electron-nuclear sublevels of the ground electronic doublet, and $\tau_{p h}(\omega)=\tau_{s} \sim 0.1 \mu \mathrm{s}$ for phonons with frequencies corresponding to transitions between the excited singlet and the ground doublet. Actually, the transitions between the electron-nuclear sublevels of the ground doublet are narrower than the transitions between the sublevels of the singlet and doublet crystal field states, and the lifetimes $\tau_{g}$ and $\tau_{s}$ may differ less than by order of magnitude. However, to fit the measured frequency dependences of the susceptibilities in the samples with higher concentrations of holmium ions, we had to diminish the factors $K_{m k}$ by $1.48(c=0.157 \%)$ and $2.7(c=0.27 \%)$ times as compared with these factors for $c=0.104 \%$. Thus, despite the increase of the concentration of the paramagnetic ions, the phonon bottleneck effect weakens due to broadening of the spectral bands of the resonant phonons and possible decrease of the phonon lifetimes.

To illustrate the phonon bottleneck effect on the relaxation rates, we present in Fig. 11(a) the calculated rates in the sample with the concentration $c=0.104 \%$ versus the magnetic field. These rates are condensed within the much narrower range as compared with the relaxation rates represented in Figs $4(a, b)$, the damping effect of the gaps at the $\Delta m=0$ crossings is partly restored due to the strong suppression of the singlet-doublet transition probabilities, but there are still no remarkable variations of the lower branches at the crossing points which may be expected in accordance with the measured field dependences of the in- and out-of-phase susceptibilities represented in Figs 5.7 .

As it is seen in Fig 12 the magnetic field dependences of $\chi^{\prime}$ and $\chi^{\prime \prime}$ obtained without phonon bottleneck and cross-relaxation effects (with electron-phonon transition probabilities only, curves 1,2) have nothing in common with the experimental data (Figs 5,7). When making use of electron-phonon transition probabilities renormalized by phonon bottleneck, we obtain the curves 3,4 showing some overlap with the measured ones (the specific humps and dips appear at the odd crossing points) but their "intensities" are lower than the experimental ones, and also there is no sign of corresponding features at even and half-integer crossings. However the results dramatically change when the cross-relaxation is taken into account: in this case all crossing points manifest themselves properly (the curves 5,6 ) and are very close to the experimental results.

The cross-relaxation rates were calculated assuming 


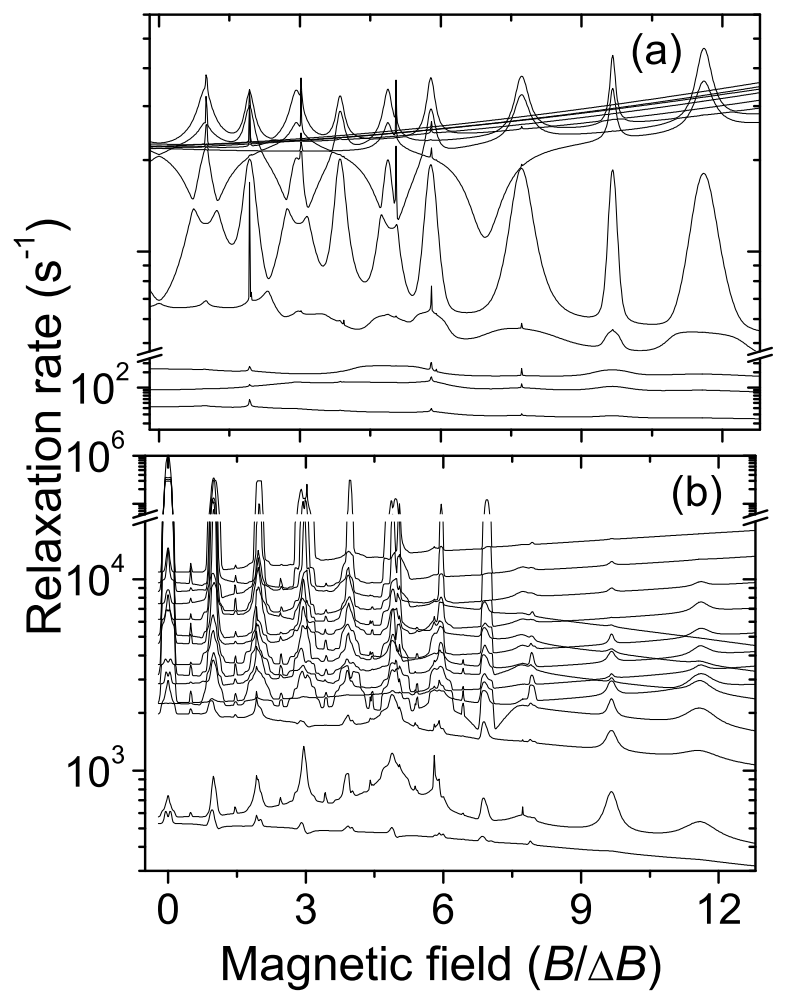

FIG. 11: The phonon bottleneck and cross relaxation effects on the relaxation rates. (a) - the relaxation rates at the temperature $2 \mathrm{~K}$ in the sample $\mathrm{LiYF}_{4}: \mathrm{Ho}^{3+}(0.104 \%)$ calculated with the renormalized electron-phonon transition probabilities due to the bottleneck effect, (b) - results of calculations with the cross relaxation terms taken into account.

the Gaussian line shape $g^{C R}=(2 \pi \Delta)^{-1} \exp \left[-\left(\omega_{p n}-\right.\right.$ $\left.\omega_{l m}\right)^{2} / 2 \Delta^{2}$ ] of the spectral density of the energy reservoir corresponding to interactions between the holmium ions. It is enough to consider in (14) only three nonzero fitting parameters, $k_{12}$ and $k_{66}$, which determine the transition probabilities within the manifold of the electronnuclear sublevels of the ground doublet, and $k_{44}$, which determines rates of the singlet-doublet transitions. For the scaling factor $\delta=2.10^{8} \mathrm{~s}^{-1}$, the fixed values of $k_{12}=k_{66}=0.1$ and $k_{44}=0.004$ independent on the concentration of the holmium ions were used in all calculations. However, the dispersion $\Delta$ of frequencies of the cross-relaxation transitions depends on the concentration, it was estimated from the EPR linewidths and corrected from a comparison of the simulated field dependences with the experimental data $(\Delta=100,120$, $140 \mathrm{MHz}$ for the $g_{44}^{C R}$ line shape, and $\Delta=185,200,240$ $\mathrm{MHz}$ for the $g_{12}^{C R}$ and $g_{66}^{C R}$ line shapes for samples with concentrations $0.104,0.157$ and $0.27 \%$, respectively). As it is seen in Fig 11(b), the cross-relaxation rates play the dominant role at all crossing points. The most important result of the cross-relaxation processes is the appearance of the low frequency $\left(10^{2}-10^{3} s^{-1}\right)$ branch in the spectrum of relaxation rates with the well resolved max-

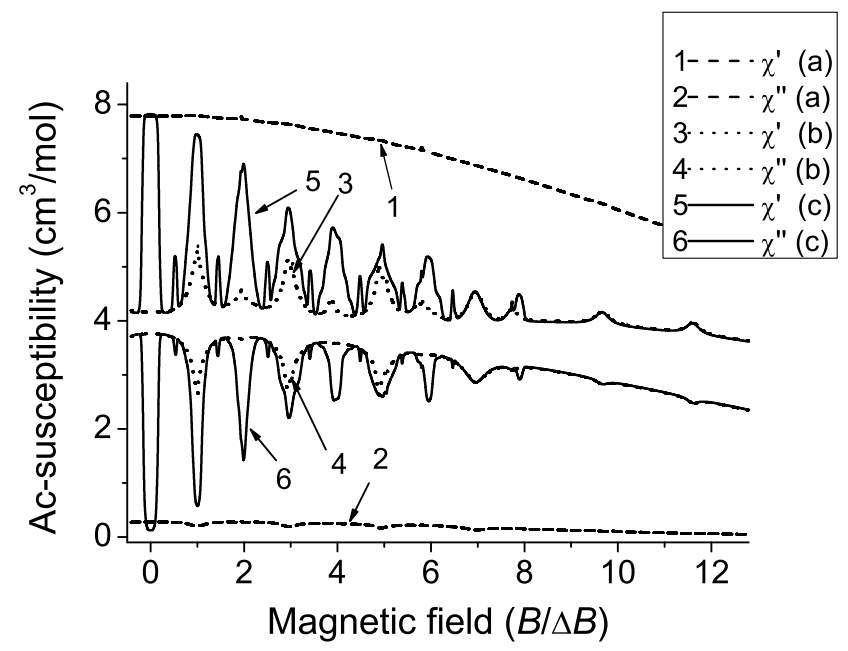

FIG. 12: The simulated ac-susceptibility of the $\mathrm{LiYF}_{4}: \mathrm{Ho}^{3+}$ $(0.27 \%)$ sample at the frequency $800 \mathrm{~Hz}$ and the temperature $2 \mathrm{~K}$, the magnetic field is declined from the c-axis by 1deg. a - the relaxation matrix contains only one-phonon transition probabilities calculated with the electron-phonon coupling constants presented in Table $1, \mathrm{~b}$ - the transition probabilities are renormalized due to the phonon bottleneck effect, c - the cross- relaxation terms are included.

ima at the crossing points. It should be noted that the calculated field dependences of the relaxation rates may change remarkably when using another cross-relaxation line shapes (the Lorentz distributions, in particular) $)^{11}$, but to analyze this problem it is necessary to perform measurements in the samples with a more wide range of concentrations of paramagnetic ions.

Small peaks at the half-integer crossings in the field dependences of $\chi^{\prime}$ (and the peaks and dips at these points in the field dependences of $\chi^{\prime \prime}$ ) are induced by the crossrelaxation singlet-doublet transitions (they disappear in the simulated curves for $k_{44}=0$ ). The value of the parameter $k_{44}$ used in simulations (the results are represented in Figs 5.57) coincides by an order of magnitude with the estimation based on the assumption of the prevailing role of the magnetic dipole-dipole interactions:

$$
k_{44}=2 \pi c a^{6} \sum\left(\frac{3 x z}{r^{5}}\right)^{2}
$$

here the sum is taken over all $Y^{3+}$ sites, $a$ is the lattice constant, and $c$ is the concentration of the impurity holmium ions. However, values of the parameters $k_{12}$ and $k_{66}$ mentioned above are much larger than the similar estimations, and it is possible that the virtual phonon exchange contributes essentially into the interaction between the holmium ions in the ground state, in particular, due to strong coupling with the dynamic lattice deformations of $B_{g}$ symmetry.

The peaks $C_{d}$ in the high-field dependences of $\chi^{\prime}$ and $\chi^{\prime \prime}$ close to the magnetic field value $B=8 \Delta B$ in Fig[ 
correspond to the anti-crossing of the electron-nuclear sublevels of the excited singlet (see Fig 4). These peaks appear due to a combined action of the random crystal field (described by the Hamiltonian $H_{S}$, see eq.(19)) and the cross-relaxation processes (singlet-doublet transitions). So, we had a possibility to determine independently the $k_{44}$ parameter from fitting intensities of $C_{h i}$ peaks, and parameters of the Hamiltonian $H_{S}$ from fitting intensities of the $C_{d}$ peaks. The best fit was achieved when the parameters $B_{2}^{2}, B_{2}^{-2}$ from Ref (see section III above) were increased by a common factor of $1.5,1.8$ and 3.3 for samples studied in this work with holmium concentrations $0.104 \%, 0.157 \%$ and $0.27 \%$, respectively, and natural abundances of $\mathrm{Li}$ isotopes. It should be noted that we have not succeeded in exact description of measured real and imaginary parts of the dynamic susceptibilities at any frequency, temperature and external magnetic field values. In particular, we obtained correct positions of the maxima in the frequency dependences of the out-of phase susceptibilities, but the calculated maximum values are higher than the measured data (see Fig.9). The discrepancies between theory and experiment in Fig.7 are the consequences of this lack of the theory. However, the specific behavior of the susceptibility in the vicinity of high field anti-crossings is reproduced by calculations as well. To derive a more elaborated model, we need additional experimental data at higher frequencies.

It follows from the simulations that an additional narrow peak should split out from the peak $C_{d}$ in the plot of $\chi^{\prime}$ versus the magnetic field (see the curve 5 in Fig 12) in the transverse magnetic field, in particular, due to a rather small misalignment of the sample. Because such a peak was not observed, we had good reasons to believe that the samples were oriented with the accuracy better than 1 degree.

Using the model parameters determined from the analysis of the frequency and field dependences of $\chi^{\prime}$ and $\chi^{\prime \prime}$, we obtained a satisfactory description of the temperature dependences as well (see Fig 10). The transformations of the magnetic field dependences of the out-of-phase susceptibility at the fixed frequency in the vicinity of the crossing points with temperature (Figs 577) or concentration (Fig 6) can be explained as a result of strong variation of relaxation rates with the magnetic field at these points. If the effective relaxation rate exceeds the frequency of the ac field, $\chi^{\prime \prime}$ has a dip, and if the relaxation rate is less than the frequency, $\chi^{\prime \prime}$ has a peak at the crossing point. The corresponding "peak to dip" transformations can be easily recognized in Figs 5.7 .7$.

\section{CONCLUSION}

We conclude that the microscopic model of a linear non-resonant response of the electron-nuclear subsystem in the highly diluted paramagnetic crystal $\mathrm{LiYF}_{4}: \mathrm{Ho}^{3+}$ on the weak ac field has been derived. The model operates with a few phenomenological parameters introduced to account for a finite heat capacity of the resonant phonons and cross-relaxation processes. The independent on temperature finite phonon lifetimes of about $1 \mu \mathrm{s}$, comparable with the phonon time of flight between the sample boundaries, have been determined from fitting the results of calculations to the measured frequency dependences of out-of-phase susceptibilities in the samples with different holmium concentrations. The parameters of the interaction between paramagnetic ions, which determine cross-relaxation rates, were obtained from fitting the simulated dependences of in-phase and out-ofphase susceptibilities on the external magnetic field. The obtained value of the parameter $\mathrm{k}_{44}$, which defines cotunneling processes at half-integer crossing points in the spectrum of a pair of $\mathrm{Ho}^{3+}$ ions, agrees with the estimation based on the dipole-dipole mechanism of inter-ion coupling. However, to present definite conclusions about the most important terms in the Hamiltonian of interaction between the holmium ions, which are responsible for the cross-relaxation processes at integer crossing and anti-crossing points, it is necessary to derive a more elaborated theory of cross-relaxation than the high temperature approach of Ref.[5].

The model containing single-ion crystal-field including random strains, electron-phonon transitions including bottleneck, hyperfine and cross-spin interactions, is comprehensive and can be used to predict the detailed magnetization dynamics in $\mathrm{LiYF}_{4}: \mathrm{Ho}^{3+}$ crystals at ultra low temperatures and at higher frequencies. Note that half-integer transitions were observed at elevated temperatures (above $1.5 \mathrm{~K}$ ) only in ac susceptibility experiments or at fast sweeping field in the low temperature (below $0.1 \mathrm{~K}$ ) magnetization measurements where the bottleneck effects stabilized an effective spin-phonon temperature of half a Kelvin [2, 3, 6]. In the present theoretical approach half-integer transitions result from resonant cross-relaxation transitions involving sublevels of the lower singlet with the activation energy of $\sim 10 \mathrm{~K}$, which is satisfactory to fit the experimental data taken at liquid helium temperatures. A more detailed analysis of the magnetization dynamics in the sweeping fields will be presented in a separate paper. The theory may be additionally tested by measurements of the ac susceptibility and NMR in a tilted magnetic field. Finally, this theoretical approach may be also expanded to other magnetic systems, in particular, the single molecule magnets.

\section{acknowledgements}

This work was supported by INTAS (project 03-514943) and the Ministry of Education and Science of Russian Federation (project RNP 2.1.1.7348). 
1 K.H. Hellwege, J. Kotzler and G. Weber, Z. Physik 217, 373 (1968).

2 R. Giraud, A.M. Tkachuk and B. Barbara, Phys. Rev. Lett. 91, 257204 (2003).

3 R. Giraud, W. Wernsdorfer, A.M. Tkachuk, D. Mailly and B. Barbara, Phys. Rev. Lett. 87, 057203 (2001).

${ }^{4}$ L. Thomas, F. Lionti, R. Ballou, D. Gatteschi, R. Sessoli and B. Barbara, Nature (London) 383, 145 (1996).

${ }^{5}$ N. Bloembergen, S. Shapiro, P.S. Pershan and J.O. Artman, Phys. Rev. 114, 445 (1959).

${ }^{6}$ B. Barbara, R. Giraud, W. Wernsdorfer, D. Mailly, P. Lejay, A. Tkachuk and H. Suzuki, J. Magn. Magn. Mat. 272276, 1024 (2004)

7 E.S. Grinberg, A.V. Duglav and B.I. Kochelaev, Zh. Eksp. Teor. Fiz. 82, 888 (1982).

8 M.J. Graf, A. Lascialfari, F. Borsa, A.M. Tkachuk and B. Barbara, Phys. Rev.B 73, 024403 (2006)

9 G.S. Shakurov, M.V. Vanyunin, B.Z. Malkin, B. Barbara, R.Yu. Abdulsabirov and S.L. Korableva, Appl. Magn. Reson. 28, 251 (2005).

10 M.N. Leuenberger and D. Loss, Phys. Rev. 61, 1286 (2000).

11 T. Pohjola and H. Schoeller, Phys. Rev. B 62, 15026 (2000).

12 P. Santini, S. Carretta, E. Liviotti, G. Amoretti, P. Carretta, M. Filibian, A. Lascialfari and E. Micotti, Phys.Rev.Lett. 94, 077203 (2005).

13 S.A. Al'tshuler and B.M. Kozirev, Electron Paramagnetic Resonance, NAUKA, Moscow, 1972

14 J.M. Baker, J. Phys. C: Solid St. Phys. 4, 1631 (1971)

15 J.H. Van-Vleck, Phys. Rev. 59, 724, 730 (1941)

16 E. Garcia and R.R. Ryan, Acta Cryst. C 49, 2053 (1993)

17 N. Karayianis and D.E. Wortman, J. Phys. Chem. Solids 37, 675 (1976)

18 N.I. Agladze and M.N. Popova, Sol. St. Commun. 55, 1097 (1985)

19 B.M. Walsh, G.W. Grew and N.P. Barnes, J. Phys.: Condens. Matter 17, 7643 (2005)
20 A.V. Vinokurov, B.Z. Malkin, A.I. Pominov and A.L. Stolov, Fiz. Tverd. Tela, 28, 381 (1986) (Sov. Phys. Solid State, 1986, 28, 211 (1986))

21 R.Yu. Abdulsabirov, A.A. Kazantsev, S.L. Korableva, B.Z. Malkin, S.I. Nikitin and A.L. Stolov. J. Lumin. 117, 225 (2006)

22 S.A. Al'tshuler, V.I. Krotov and B.Z. Malkin, Pis'ma ZETF, 32, 232 (1980) (Sov. Phys. JETP Lett., 32, 214 (1980))

23 L.A. Bumagina, V.I. Krotov, B.Z. Malkin and A.Kh. Khasanov, Zh. Eksp. Teor. Fiz., 80, 1543 (1981) (Sov. Phys. JETP, 53, 792 (1981))

24 J.P.D. Martin, T. Boonyarith and N.B. Manson, J. Lumin. 63, 297 (1995)

25 S. Salaun, A. Bulou, M. Rousseau, B Hennion and J.Y. Gesland, J. Phys. Condens. Matter 9, 6957 (1997)

26 B.Z. Malkin in: Spectroscopy of solids containing rareearth ions, ed. by A.A.Kaplyanskii and R.M.Macfarlane, Elsevier Science Publishers,Amsterdam, 1987, ch. 2, p. 1349.

27 P. Erdos and J.H. Kang, Phys. Rev. B 6, 3393 (1972)

28 A.J. Freeman and R.E. Watson, Phys. Rev. 127, 2058 (1962)

29 O.J. Sovers, J. Phys. Chem. Solids 28, 1073 (1967)

30 E. Clementi and A.D. McLean, Phys. Rev. A 133, 419 (1964)

31 A.A. Antipin, L.A. Bumagina, B.Z. Malkin and R.M. Rakhmatullin, Fiz. Tverd. Tela (Leningrad) 23, 2700 (1981) (Sov. Phys. Solid State 23, 1583 (1981))

32 P. Blanchfield and G.A. Saunders, J. Phys. C 12, 4673 (1979)

33 D.H. Reich, T.F. Rosenbaum and G. Aeppli, and H.J. Guggenheim, Phys. Rev. 34, 4956 (1986).

34 D.H. Reich, T.F. Rosenbaum, and G. Aeppli, Phys. Rev. Lett. 59, 1969 (1987).

35 D.H. Reich, B. Ellman, J. Yang, T.F. Rosenbaum, G. Aeppli, and D.P. Belanger, Phys. Rev. 42, 4631 (1990). 